DAMTP-2003-49 MIFP-03-12 UPR/0852-T

June 2003, hep-th/0306043

\title{
Consistent Group and Coset Reductions of the Bosonic String
}

\author{
M. Cvetič ${ }^{\dagger}$, G.W. Gibbons ${ }^{\star}$, H. Lü ${ }^{\ddagger}$ and C.N. Pope ${ }^{\ddagger}$ \\ $\dagger$ School of Natural Sciences, \\ Institute for Advanced Studies, Princeton, NJ 08540, USA \\ *DAMTP, Centre for Mathematical Sciences, Cambridge University, \\ Wilberforce Road, Cambridge CB3 OWA, UK \\ ${ }^{\ddagger}$ George P. $\&$ Cynthia W. Mitchell Institute for Fundamental Physics, \\ Texas A\&M University, College Station, TX 77843-4242, USA
}

\begin{abstract}
$\underline{\text { ABSTRACT }}$
Dimensional reductions of pure Einstein gravity on cosets other than tori are inconsistent. The inclusion of specific additional scalar and $p$-form matter can change the situation. For example, a $D$-dimensional Einstein-Maxwell-dilaton system, with a specific dilaton coupling, is known to admit a consistent reduction on $S^{2}=S U(2) / U(1)$, of a sort first envisaged by Pauli. We provide a new understanding, by showing how an $S^{3}=S U(2)$ group-manifold reduction of $(D+1)$-dimensional Einstein gravity, of a type first indicated by DeWitt, can be broken into in two steps; a Kaluza-type reduction on $U(1)$ followed by a Pauli-type coset reduction on $S^{2}$. More generally, we show that any $D$-dimensional theory that itself arises as a Kaluza $U(1)$ reduction from $(D+1)$ dimensions admits a consistent Pauli reduction on any coset of the form $G / U(1)$. Extensions to the case $G / H$ are given. Pauli coset reductions of the bosonic string on $G=(G \times G) / G$ are believed to be consistent, and a consistency proof exists for $S^{3}=S O(4) / S O(3)$. We examine these reductions, and arguments for consistency, in detail. The structures of the theories obtained instead by DeWitt-type group-manifold reductions of the bosonic string are also studied, allowing us to make contact with previous such work in which only singlet scalars are retained. Consistent truncations with two singlet scalars are possible. Intriguingly, despite the fact that these are not supersymmetric models, if the group manifold has dimension 3 or 25 they admit a superpotential formulation, and hence first-order equations yielding domain-wall solutions.
\end{abstract}




\section{Contents}

1 Introduction 2

2 The Bosonic String $\quad 6$

3 DeWitt Reduction of the Bosonic String $\quad 8$

3.1 Reduction of the metric . . . . . . . . . . . . . . . 8

3.2 Reduction of the 3 -form field . . . . . . . . . . . . . . . . . 10

3.3 The Lagrangian and gauge symmetries . . . . . . . . . . . . . . . . . . . 12

4 Consistent Truncation of the Scalar Moduli 13

5 DeWitt $=$ Pauli $\circ$ Kaluza $\quad 14$

5.1 The case $S U(2) / U(1) \ldots \ldots \ldots \ldots \ldots \ldots \ldots$

5.2 The case $G / U(1) \ldots \ldots \ldots \ldots \ldots \ldots$

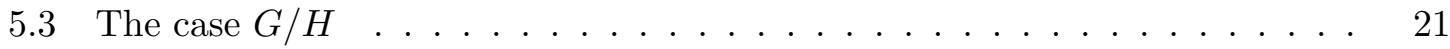

6 Pauli Reductions of the Bosonic String 22

6.1 Introduction . . . . . . . . . . . . . . . . . . 22

6.2 Pauli reductions of the bosonic string on $S^{3}$ and group manifolds . . . . . 24

7 Superpotentials and Solutions $\quad 27$

7.1 Superpotentials for the truncated system . . . . . . . . . . . . 27

7.2 Brane solutions from superpotentials . . . . . . . . . . . . . . 28

7.3 Branes without superpotentials . . . . . . . . . . . . . 30

8 Discussion $\quad 32$

A DeWitt $=$ Pauli $\circ$ DeWitt: Reductive Coset Reductions 38

B Further Examples of Coset Reductions $\quad 40$

B.1 The case of $(G \times G) / G \ldots \ldots \ldots \ldots \ldots \ldots$

B.2 The case of $S^{n}=S O(n+1) / S O(n) \ldots \ldots \ldots \ldots 42$ 


\section{Introduction}

Dimensional reductions, sometimes referred to as Kaluza-Klein reductions, have had a long and convoluted history. Shortly after the inception of general relativity, Kaluza in 1919 pointed out that by considering a circle reduction of five-dimensional Einstein gravity, one obtains a unification of gravity with Maxwell theory in four dimensions, at the cost of introducing an extra scalar field [1]. In subsequent developments this scalar field was viewed as somewhat of an embarrassment, and it was usually arbitrarily (and incorrectly) set to a constant value. This was done, for example, in the work of Klein [2] (he did, however, make the important observation that the charges are quantised). In fact it was not until the much later work of Jordan (1947) and Thiry (1948) that it was fully appreciated that if one imposes the five-dimensional Einstein equations, subject solely to the "cylindrical condition" then if the Maxwell field is non-vanishing it is dynamically inconsistent to impose the condition that the scalar is constant $[3,4]$.

The next development came with the work of Pauli in 1953, who attempted in an unpublished work to obtain $S U(2)$ Yang-Mills fields from a reduction scheme in which a sixdimensional spacetime is taken to be the product of a 2 -sphere (with its round metric) and a four-dimensional spacetime [5]. ${ }^{1}$ Nowadays, generalisations of this model are in widespread use, and are often referred to as "coset reductions." In this paper we shall call them "Pauli Reductions." Pauli realised that there are considerable problems in obtaining the Yang-Mills field equations from the six-dimensional Einstein equations in a fully consistent dynamical way. He seems to have realised, for example, that there was no justification for substituting his ansatz into the six-dimensional action functional. This point has often been overlooked in subsequent developments.

It appears that it was DeWitt in 1963 who was the first to obtain the Yang-Mills equations in $n$ dimensions from a reduction scheme in which one assumes that $(n+q)$ dimensional spacetime is invariant under the action of a compact semi-simple Lie group $G$ of dimension $q[10]$. Such reductions are frequently referred to as group-manifold reductions; in this paper we shall call them "DeWitt Reductions." In the exercise in [10], it was assumed that the metric on the orbit space of the group $G$ was the bi-invariant metric, but DeWitt

\footnotetext{
${ }^{1}$ An examination of Klein's 1938 paper on Yang-Mills gauge invariance reveals that, as far as gravity is concerned, he definitely did not have in mind a reduction either on a group manifold or a coset space. His higher-dimensional spacetime was only five-dimensional, and his metric appears to be non-commutative $[8,9,7]$. Thus although Klein made pioneering contributions to non-Abelian gauge theories, the gauge bosons did not originate from higher dimensional symmetries.
} 
indicated that a fully consistent reduction would involve taking a metric which was merely left-invariant. This results in $\frac{1}{2} q(q+1)$ scalar fields propagating in the lower-dimensional spacetime. Indeed these are the analogues of the scalar field that was often omitted in the older work on five-dimensional theories, and again one can easily see that their omission is inconsistent with the higher-dimensional vacuum Einstein equations. These fields, which parameterise the shape of the "internal" group manifold, are often referred to as "scalar moduli." It is a curious fact that if one takes the higher-dimensional theory to be not pure Einstein gravity but rather the low-energy effective action of the bosonic string, then a DeWitt reduction exists in which the scalar moduli can be consistently omitted [11].

In 1968 Kerner, apparently unaware of DeWitt's work, showed explicitly that by substituting the "restricted DeWitt ansatz" (i.e. without the scalar moduli) into the higherdimensional Einstein action one obtains a lower-dimensional action for gravity plus YangMills fields [12]. This calculation is of course correct in so far as it goes, but ignores the "consistency issue," which is whether solutions of the equations of motion of the lowerdimensional action do actually provide solutions of the higher-dimensional Einstein equations. In fact in this case they do not. Later, the theory of consistent DeWitt reductions (i.e. including the scalar moduli) was developed by Cho and Freund (1975). Further work by Scherk and Schwarz (1979) emphasised the importance of requiring that the group $G$ be unimodular (meaning that the structure constants satisfy $\left.f_{\alpha \beta}^{\alpha}=0\right)[13,14]$. The condition of unimodularity is automatically satisfied for compact Lie groups, which in practice is where our interest lies.

Somewhat earlier than the work of Cho and Freund and of Scherk and Schwarz, it was realized by Hawking (1969) [15] working on homogeneous Bianchi cosmology, i.e. DeWitt reductions to one spacetime dimension, that substitution of the ansatz into the Einstein action will not always give the correct field equations. Roughly speaking, the point is that to obtain the the field equations, an integration by parts is required. If the internal space is closed, i.e. compact without boundary, this presents no obvious problem. However if the internal space is non-compact, since all variations are, according to the ansatz, $G$-invariant, one cannot merely assume, as one ordinarily does, that they vanish outside a compact set or "at infinity." Following a period of confusion, the situation was clarified by Sneddon (1976) [16], who showed in detail that if the group $G$ is unimodular (known as class A in the Bianchi classification), then no problem arises and the procedure works. However, if the group is non-unimodular (known as class B in the Bianchi classification) then problems can and do arise and incorrect equations of motion are obtained. A simple definition of unimodular is 
that the adjoint representation of the group has unit determinant, or infinitesimally that $f_{\alpha \beta}^{\alpha}=0$. An equivalent definition is that any left-invariant measure on $G$ is also rightinvariant. Thus all top-dimensional forms on $G$ are proportional up a factor which does not depend upon the coordinates on $G$. Yet another way of stating the condition is that the generators of right translations, that is the left-invariant vector fields, have vanishing divergence. A semi-simple group (compact or not) is necessarily unimodular and a compact group is necessarily unimodular and so perhaps because they limited their attention to that case, Cho and Freund encountered no difficulty. Scherk and Schwarz by contrast considered more general groups and drew attention, apparently unaware of the work on Bianchi cosmology, to the need for unimodularity. In the context of dimensional reduction one is almost always interested in compact internal spaces, whether group manifolds or not, and so integration by parts will then be a valid manoeuvre.

The situation for Pauli reductions, i.e. coset reductions, is considerably more subtle than that for DeWitt reductions, and to date there exist very few known examples of such reductions that are consistent. Of course, if we assumed that the higher-dimensional spacetime were invariant under the action of a Lie group $G$ acting on a coset $K=G / H$, then consistency would be expected. ${ }^{2}$ However, the aim of the exercise is to obtain fields in the lower-dimensional spacetime that include all of the gauge bosons of the isometry group $G$. In order to do this, it is necessary to make an ansatz for the higher-dimensional metric and other fields that is not invariant under the action of $G$. On the other hand, we are obviously not interested in going to the opposite extreme, by retaining all the fields in a generalised Fourier expansion, which would certainly guarantee consistency, but would have little point since it would merely provide a clumsy description of an intrinsically higher-dimensional situation. Thus an important ingredient when considering a coset reduction could be said to be that one requires a reduction to a finite set of lower-dimensional fields that includes all the gauge bosons associated with the isometry group of the coset. Having established the desiderata for the ansatz, the statement of consistency is as follows: Substitution of the reduction ansatz into the higher-dimensional equations of motion must yield a set of equations of motion expressed entirely in terms of the lower-dimensional fields. In other words, if $x^{\mu}$ are coordinates on the lower-dimensional spacetime, and $y^{m}$ are coordinates on the internal space, then substituting the reduction ansatz into the higher-dimensional

\footnotetext{
${ }^{2}$ In this paper, all cosets will be what is customarily (but not completely universally) called right cosets, i.e. we quotient $G$ by the equivalence relation $g_{1} \equiv g_{2}$ if and only if there is an $h \in H$ such that $g_{1}=h g_{2}$. Thus right cosets admit global right actions of the group $G$, but in general no left action.
} 
equations of motion should result in a system of equations in which all $y^{m}$ dependence has cancelled. These lower-dimensional equations may or may not be derivable from a lowerdimensional action principle, which itself may or may not be obtained by substitution of the ansatz into the higher-dimensional action. It should be emphasised, however, that the test of consistency is determined solely by the equations of motion, and is quite independent of any considerations of action principles.

At present there is no known algorithmic prescription for obtaining consistent Pauli reduction ansätze. A systematic understanding of the conditions under which such reductions are possible is still lacking. In the few examples where consistent Pauli reductions are known, there has hitherto been little conceptual understanding of why they work beyond the bald statement that detailed calculation shows that they do.

The first two candidates yielding consistent Pauli reductions in fact arose in the compactification of eleven-dimensional supergravity. The first was the $S^{7}$ reduction to fourdimensional $S O(8)$ gauged $N=8$ supergravity, for which a proof of consistency was presented in [17]; and the second was the $S^{4}$ reduction to $S O(5)$-gauged $N=4$ supergravity in seven dimensions, for which the consistency proof was presented in [18]. It is widely believed that a consistent Pauli reduction of type IIB supergravity on $S^{5}$ also exists, which would yield $S O(6)$-gauged $N=8$ supergravity in five dimensions. A consistent $S^{5}$ Pauli reduction of a truncation of type IIB supergravity to its $S L(2, \mathbb{R})$-singlet sector, yielding a five-dimensional theory with all the gauge bosons of $S O(6)$, has been constructed in [19]. Various explicit consistent reductions yielding subsets of the maximally-supersymmetric cases have also been constructed (see, for example, [20, 21, 22, 23]).

The consistent Pauli reductions mentioned above might lead one to conclude that supersymmetry was an essential ingredient in the consistency of the Pauli reduction. However, various further examples of consistent Pauli reductions exist which lie entirely outside the framework of supergravity. They do still, however, require that one consider a higherdimensional theory that goes beyond pure Einstein gravity. Two such theories were considered in [24], one being the low-energy effective action for the $D$-dimensional bosonic string, and the other being a specific $D$-dimensional theory of Einstein gravity coupled to a Maxwell field and a dilaton. It was shown that a consistent Pauli reduction of the $D$-dimensional bosonic string on $S^{3}$ or $S^{D-3}$ is always possible, and that a consistent Pauli reduction of the $D$-dimensional Einstein-Maxwell-dilaton theory on $S^{2}$ is always possible [24]. It should be emphasised that the $S^{3}$ reduction of the bosonic string is of Pauli type, and not merely of DeWitt type; it yields the full set of $S O(4)$ gauge fields. This latter example lends support 
to an old conjecture in [25], asserting that a consistent Pauli reduction of the bosonic string on the group manifold $G$ should always be possible; again, keeping the full set of gauge bosons of the $G \times G$ isometry group of the bi-invariant metric on $G$.

\section{The Bosonic String}

A bosonic string moving in background fields $G_{M N}, B_{M N}, \Phi$ in $D$ dimensions may be regarded as a generalized 2-dimensional non-linear sigma model. The demand that the beta functions vanish gives rise to the equations of motion for the background fields and these equations of motion may in fact be derived from a stationary action principle. To lowest order in the inverse string tension $\frac{1}{2 \pi \alpha^{\prime}}$, one has [26]

$$
\begin{aligned}
16 \pi^{2} \beta^{\Phi} & =\frac{D-26}{3 \alpha^{\prime}}+\left[4(\nabla \Phi)^{2}-4 \nabla^{2} \Phi-R+\frac{1}{12} H^{2}\right] \\
\beta_{M N}^{G} & =R_{M N}-\frac{1}{4} H_{M}{ }^{P Q} H_{N P Q}+s \nabla_{M} \nabla_{N} \Phi \\
\beta_{M N}^{B} & =\nabla_{P} H^{P}{ }_{M N}-2 H_{M N}^{P} \nabla_{P} \Phi
\end{aligned}
$$

where the 3 -form $H_{M N P}=3 \partial_{[M} B_{N P]}$ is locally exact and hence closed

$$
\partial_{[Q} H_{M N P]}=0
$$

The covariant derivative $\nabla$ defines the usual metric-preserving torsion-free Levi-Civita affine connection of the string metric $G_{M N}$. It is often convenient to re-express them in terms of another metric-preserving affine connection whose torsion is given by the 3 -form $H_{M N P}$. This connection we call $\nabla^{+}$, and acting on a vector field $V^{M}$ we have

$$
\nabla_{M}^{+} V^{N}=\nabla_{M} V^{N}+\frac{1}{2} H_{N}{ }^{N}{ }_{C} V^{C}
$$

The torsion $T_{M}{ }^{N}{ }_{P}=H_{M}{ }^{N}{ }_{P}$. Acting on a scalar such as the dilaton $\Phi, \nabla^{+}$reduces, as does $\nabla$, to the partial derivative

$$
\nabla_{M}^{+} \Phi=\partial_{M} \Phi
$$

However, the "Hessian" is not symmetric; in fact

$$
2 \nabla_{[M}^{+} \nabla_{N]}^{+} \Phi=H_{M}{ }^{P}{ }_{N} \partial_{P} \Phi
$$

Associated with $\nabla^{+}$in the usual way we have its curvature tensor $R^{+A}{ }_{B M N}=-R_{B N M}^{A}$ and its Ricci tensor $R_{B N}^{+}=R_{B A N}^{+A}$, which, in the presence of torsion, ceases to be 
symmetric. We define the associated scalar curvature by $R^{+}=G^{M N} R_{M N}^{+}$. The last two equations of motion in (2.1) now get combined into the statement that

$$
R_{M N}^{+}+2 \nabla_{M}^{+} \nabla_{N}^{+} \Phi=0
$$

The first equation becomes

$$
4\left(\nabla^{+} \Phi\right)^{2}-4\left(\nabla^{+}\right)^{2} \Phi-R^{+}-\frac{1}{6} H^{2}+\frac{D-26}{48 \pi^{2}}=0 .
$$

In addition one must bear in mind the torsion 3-form is closed. This latter fact renders writing down an action principle in terms of the string metric and the components of the connection $\Gamma^{+}{ }_{M}{ }^{N}{ }_{P}$ rather difficult. However one merit of this formulation is that it makes it easy to see that the metric product of two solutions is also a solution, as long as the central charges can be balanced. It also makes it immediate that the equations of motion are solved by a constant dilaton $\Phi$, with the metric a product $\mathbb{E}^{p, 1} \times G$, where $G$ is itself a product of semi-simple groups carrying their Killing metrics and where $\nabla^{+}$is taken to be the flat parallelizing connection obtained by right or left translation on each factor group. We shall encounter such solutions latter in the paper. We shall refer to such backgrounds as WZWN ground states.

In the string conformal frame, the action whose equations of motion imply the vanishing of the beta functions $(2.1)$ is ${ }^{3}$

$$
\mathcal{L}=e^{-2 \Phi}\left(R * \mathbb{1}+4 * d \Phi \wedge d \Phi-\frac{1}{2} * H_{(3)} \wedge H_{(3)}\right) .
$$

One can also pass to the Einstein conformal frame. Setting

$$
d s_{\text {string }}^{2}=e^{-\frac{1}{2} a \phi} d s_{\text {Einstein }}^{2}, \quad \Phi=-\frac{1}{a} \phi,
$$

where $a^{2}=8 /(D-2)$, gives the bosonic string Lagrangian in the standard normalisation, in the Einstein frame. Since we shall be performing dimensional reductions of this theory in subsequent sections, we shall present it here with hats put on all the quantities in $D$ dimensions:

$$
\hat{\mathcal{L}}=\hat{R} \hat{*} \mathbb{1}-\frac{1}{2} \hat{*} d \hat{\phi} \wedge d \hat{\phi}-\frac{1}{2} e^{\hat{a} \hat{\phi}} \hat{*} \hat{G}_{(3)} \wedge \hat{G}_{(3)},
$$

where $\hat{G}_{(3)}=d \hat{B}_{(2)}$, and the constant $\hat{a}$ is given by

$$
\hat{a}^{2}=\frac{8}{D-2} .
$$

\footnotetext{
${ }^{3}$ Since, in the special case of ten dimensions, this is the same action as in the NS-NS sector of superstring theory, all the subsequent discussion of the bosonic string reductions applies also to the NS-NS sector of superstring theory.
} 
The group manifold reduction, which we refer to as the "DeWitt reduction," can be viewed as a general harmonic expansion on a group manifold $G$ of dimension $q$, in which all the lower-dimensional fields that are associated with harmonics that are singlets under the left action $G_{L}$ of the group $G$ are retained, whilst all fields associated with non-singlet harmonics are set to zero. This truncation yields just a finite number of $n$-dimensional fields, since $G_{L}$ acts transitively on $G$. Crucially, the truncation is necessarily a consistent one, meaning that setting the non-singlet fields to zero is consistent with their own equations of motion. The essential point here is that despite the non-linearity of the full system of equations, nonlinear products of the retained $G_{L}$ singlets can obviously never generate $G_{L}$ non-singlets, and thus the retained fields cannot act as sources for the fields that are set to zero.

\section{DeWitt Reduction of the Bosonic String}

Let $\sigma^{\alpha}$ denote a set of left-invariant 1-forms on the $q$-dimensional group manifold $G$; they satisfy

$$
d \sigma^{\alpha}=-\frac{1}{2} f_{\beta \gamma}^{\alpha} \sigma^{\beta} \wedge \sigma^{\gamma}
$$

where $f_{\beta \gamma}^{\alpha}$ are the structure constants of the Lie algebra of $G$. We shall assume throughout that $G$ is compact and semisimple, and so the Cartan-Killing metric

$$
g_{\alpha \beta} \equiv-\frac{1}{2} f_{\delta \alpha}^{\gamma} f_{\gamma \beta}^{\delta}
$$

is non-degenerate, and positive-definite. It follows from the Jacobi identity $f_{\lambda[\alpha}^{\delta} f_{\beta \gamma]}^{\lambda}=0$ that if $g_{\alpha \beta}$ is used to lower the upper index on the structure constants, the resulting tensor $f_{\alpha \beta \gamma} \equiv g_{\alpha \delta} f_{\beta \gamma}^{\delta}$ is totally antisymmetric.

\subsection{Reduction of the metric}

We consider the standard DeWitt ansatz ${ }^{4}$ for reducing the $D=n+q$ dimensional metric $d \hat{s}^{2}$

$$
d \hat{s}^{2}=e^{2 \alpha \varphi} d s^{2}+g^{-2} e^{2 \beta \varphi} h_{\alpha \beta} \nu^{\alpha} \nu^{\beta},
$$

\footnotetext{
${ }^{4}$ It should, perhaps, be remarked that the term "ansatz" is often, as here, used inappropriately when describing dimensional reduction procedures, since it carries the connotation that a trial substitution that might or might not be successful is being attempted. In fact in Kaluza $S^{1}$ reductions and DeWitt groupmanifold reductions there is only one possible "ansatz" and there is no possibility of failure; the "reduction ansatz" is nothing but an appropriate parameterisation of the group-invariant higher-dimensional fields in terms of lower-dimensional ones. Since there seems to be no other satisfactory word that succinctly expresses the true nature of the procedure, we shall perpetuate the use of the term "ansatz" despite its inappropriateness.
} 
where

$$
\nu^{\alpha} \equiv \sigma^{\alpha}-g A^{\alpha}
$$

Here $d s^{2}$ is the reduced $n$-dimensional metric, $A^{\alpha}$ are the Yang-Mills potentials for the gauge group $G$, and $h_{\alpha \beta}$ is a unimodular symmetric matrix parameterising the scalar degrees of freedom. The Yang-Mills field strengths are given by

$$
F^{\alpha}=d A^{\alpha}+\frac{1}{2} g f_{\beta \gamma}^{\alpha} A^{\beta} \wedge A^{\gamma}
$$

The constants $\alpha$ and $\beta$ are chosen to be given by

$$
\alpha=-\sqrt{\frac{q}{2(n-2)(n+q-2)}}, \quad \beta=-\frac{\alpha(n-2)}{q} .
$$

These choices ensure that the reduction of the Einstein-Hilbert action yields a pure EinsteinHilbert term in $n$ dimensions, with no prefactor involving the breathing-mode scalar $\varphi$, and that $\varphi$ has a canonically-normalised kinetic term in $n$ dimensions.

We shall choose the vielbein basis

$$
\hat{e}^{a}=e^{\alpha \varphi} e^{a}, \quad \hat{e}^{i}=g^{-1} e^{\beta \varphi} \Phi_{\alpha}^{i} \nu^{\alpha}
$$

where

$$
h_{\alpha \beta}=\Phi_{\alpha}^{i} \Phi_{\beta}^{i} .
$$

We also introduce the covariant exterior derivative $D$, whose action on the 1 -forms $\nu^{\alpha}$ is given by

$$
D \nu^{\alpha} \equiv d \nu^{\alpha}+g f_{\beta \gamma}^{\alpha} A^{\beta} \wedge \nu^{\gamma}=-g F^{\alpha}-\frac{1}{2} f_{\beta \gamma}^{\alpha} \nu^{\beta} \wedge \nu^{\gamma}
$$

From these expressions, we find that

$$
\begin{aligned}
d \hat{e}^{a}= & -\alpha e^{-\alpha \phi} \partial_{b} \phi \hat{e}^{a} \wedge \hat{e}^{b}-\omega^{a}{ }_{b} \wedge \hat{e}^{b}, \\
d \hat{e}^{i}= & e^{-\alpha \phi}\left(\Phi^{-1}\right)_{j}^{\alpha}\left(D_{a} \Phi_{\alpha}^{i}\right) \hat{e}^{a} \wedge \hat{e}^{j}+\beta e^{-\alpha \phi} \partial_{a} \phi \hat{e}^{a} \wedge \hat{e}^{i}-\frac{1}{2} e^{(\beta-2 \alpha) \phi} F_{a b}^{i} \hat{e}^{a} \wedge \hat{e}^{b} \\
& -\frac{1}{2} g e^{-\beta \phi} \Phi_{\alpha}^{i}\left(\Phi^{-1}\right)_{j}^{\beta}\left(\Phi^{-1}\right)_{k}^{\gamma} f^{\alpha}{ }_{\beta \gamma} \hat{e}^{j} \wedge \hat{e}^{k},
\end{aligned}
$$

where we have defined $F_{a b}^{i} \equiv \Phi_{\alpha}^{i} F_{a b}^{\alpha}$. The torsion-free spin connection $\hat{\omega}_{B}^{A}$, defined by $d \hat{e}^{A}=-\hat{\omega}^{A}{ }_{B} \wedge \hat{e}^{B}$ and $\hat{\omega}_{A B}=-\hat{\omega}_{B A}$, turns out to be

$$
\begin{aligned}
\hat{\omega}_{a b}= & \omega_{a b}+\alpha e^{-\alpha \phi}\left(\partial_{b} \phi \eta_{a c} \hat{e}^{c}-\partial_{a} \phi \eta_{b c} \hat{e}^{c}\right)+\frac{1}{2} e^{(\beta-2 \alpha) \phi} F_{a b}^{i} \hat{e}^{i}, \\
\hat{\omega}_{a i}= & -e^{-\alpha \phi} P_{a i j} \hat{e}^{j}-\beta e^{-\alpha \phi} \partial_{a} \phi \hat{e}^{i}+\frac{1}{2} e^{(\beta-2 \alpha) \phi} F_{a b}^{i} \hat{e}^{b}, \\
\hat{\omega}_{i j}= & e^{-\alpha \phi} Q_{a i j} \hat{e}^{a} \\
& +\frac{1}{2} g e^{-\beta \phi}\left[\Phi_{\alpha}^{k}\left(\Phi^{-1}\right)_{i}^{\beta}\left(\Phi^{-1}\right)_{j}^{\gamma}+\Phi_{\alpha}^{j}\left(\Phi^{-1}\right)_{i}^{\beta}\left(\Phi^{-1}\right)_{k}^{\gamma}-\Phi_{\alpha}^{i}\left(\Phi^{-1}\right)_{j}^{\beta}\left(\Phi^{-1}\right)_{k}^{\gamma}\right] f_{\beta \gamma}^{\alpha} \hat{e}^{k},
\end{aligned}
$$


where

$$
P_{a i j} \equiv \frac{1}{2}\left[\left(\Phi^{-1}\right)_{i}^{\alpha} D_{a} \Phi_{\alpha}^{j}+\left(\Phi^{-1}\right)_{j}^{\alpha} D_{a} \Phi_{\alpha}^{i}\right], \quad Q_{a i j} \equiv \frac{1}{2}\left[\left(\Phi^{-1}\right)_{i}^{\alpha} D_{a} \Phi_{\alpha}^{j}-\left(\Phi^{-1}\right)_{j}^{\alpha} D_{a} \Phi_{\alpha}^{i}\right] .
$$

Defining

$$
\hat{\omega}_{A B} \equiv \omega_{C A B} \hat{e}^{C}, \quad \omega_{A} \equiv \eta^{B C} \hat{\omega}_{B C A},
$$

the $(n+q)$-dimensional Einstein Hilbert Lagrangian $L=\hat{e} \hat{R}$ can be written, up to an irrelevant total derivative, as

$$
L=\hat{\mathrm{e}}\left(\hat{\omega}_{A B C} \hat{\omega}^{C A B}+\hat{\omega}^{A} \hat{\omega}_{A}\right),
$$

From this, one can straightforwardly determine using (3.11) that after reduction on the group manifold $G$ the $(n+q)$-dimensional Einstein-Hilbert action leads to an action in $n$ dimensions given in terms of the Lagrangian $n$-form ${ }^{5}$

$$
\begin{aligned}
\mathcal{L}_{\mathrm{EH}}= & R * \mathbb{1}-\frac{1}{2} * d \varphi \wedge d \varphi-* P_{i j} \wedge P_{i j}-\frac{1}{2} e^{2(\beta-\alpha) \varphi} h_{\alpha \beta} * F^{\alpha} \wedge F^{\beta} \\
& -\frac{1}{4} g^{2} e^{2(\alpha-\beta) \varphi}\left(h_{\alpha \beta} h^{\gamma \delta} h^{\rho \sigma} f_{\gamma \rho}^{\alpha} f^{\beta}{ }_{\delta \sigma}+2 h^{\alpha \beta} f_{\delta \alpha}^{\gamma} f^{\delta}{ }_{\gamma \beta}\right) * \mathbb{1} .
\end{aligned}
$$

\subsection{Reduction of the 3 -form field}

We implement the group-manifold reduction at the level of the 2 -form potential $\hat{B}_{(2)}$, by writing

$$
\hat{B}_{(2)}=m g^{-3} \omega_{(2)}+B_{(2)}+g^{-1} B_{(1) \alpha} \wedge \nu^{\alpha}+\frac{1}{2} g^{-2} \chi_{\alpha \beta} \nu^{\alpha} \wedge \nu^{\beta}
$$

where $\omega_{(2)}$ is such that

$$
d \omega_{(2)}=\frac{1}{6} f_{\alpha \beta \gamma} \sigma^{\alpha} \wedge \sigma^{\beta} \wedge \sigma^{\gamma} .
$$

We also define lower-dimensional field strengths, by writing

$$
\hat{G}_{(3)} \equiv d \hat{B}_{(2)}=G_{(3)}+g^{-1} G_{(2) \alpha} \wedge \nu^{\alpha}+\frac{1}{2} g^{-2} G_{(1) \alpha \beta} \wedge \nu^{\alpha} \wedge \nu^{\beta}+\frac{1}{6} g^{-3} G_{(0) \alpha \beta \gamma} \nu^{\alpha} \wedge \nu^{\beta} \wedge \nu^{\gamma} .
$$

It follows, therefore, that we shall have

$$
\begin{aligned}
G_{(3)} & =d B_{(2)}+B_{(1) \alpha} \wedge F^{\alpha}+\frac{1}{6} m f_{\alpha \beta \gamma} A^{\alpha} \wedge A^{\beta} \wedge A^{\gamma}, \\
G_{(2) \alpha} & =D B_{(1) \alpha}+\chi_{\alpha \beta} F^{\beta}+\frac{1}{2} m f_{\alpha \beta \gamma} A^{\beta} \wedge A^{\gamma}, \\
G_{(1) \alpha \beta} & =D \chi_{\alpha \beta}+g f_{\alpha \beta}^{\gamma} B_{(1) \gamma}+m f_{\gamma \alpha \beta} A^{\gamma}, \\
G_{(0) \alpha \beta \gamma} & =m f_{\alpha \beta \gamma}-3 g \chi_{\delta[\alpha} f_{\beta \gamma]}^{\delta} .
\end{aligned}
$$

\footnotetext{
${ }^{5}$ Substitution of a Kaluza or DeWitt reduction ansatz into a higher-dimensional Lagrangian is always a valid procedure, provided that all the appropriate group-invariant fields are included, and that the group is unimodular. The resulting lower-dimensional Lagrangian yields the same equations of motion as those that would result from substitution of the ansatz into the higher-dimensional equations of motion.
} 
It is convenient at this stage to introduce redefined quantities as follows:

$$
\begin{aligned}
\widetilde{\chi}_{\alpha} & \equiv \frac{1}{2} f_{\alpha}^{\beta \gamma} \chi_{\beta \gamma}, \quad \tilde{\chi}_{\alpha \beta} \equiv \chi_{\alpha \beta}-f_{\alpha \beta}^{\gamma} \tilde{\chi}_{\gamma}, \\
\widetilde{B}_{(1) \alpha} & \equiv B_{(1) \alpha}+m g^{-1} A_{\alpha}+g^{-1} D \widetilde{\chi}_{\alpha}, \\
\widetilde{B}_{(2)} & \equiv B_{(2)}-g^{-1} \widetilde{\chi}_{\alpha} F^{\alpha} \\
\widetilde{G}_{(2) \alpha} & \equiv G_{(2) \alpha}+m g^{-1} F_{\alpha}, \\
\tilde{\omega}_{(2)} & \equiv \omega_{(2)}-g A_{\alpha} \wedge \nu^{\alpha},
\end{aligned}
$$

where we have defined $F_{\alpha} \equiv g_{\alpha \beta} F^{\beta}$. We also shift $\hat{B}_{(2)}$ by adding to it the total derivative $d\left(-g^{-2} \tilde{\chi}_{\alpha} \nu^{\alpha}\right)$. In terms of the new variables, the ansatz for the potential becomes

$$
\hat{B}_{(2)}=m g^{-3} \tilde{\omega}_{(2)}+\widetilde{B}_{(2)}+g^{-1} \widetilde{B}_{(1) \alpha} \wedge \nu^{\alpha}+\frac{1}{2} g^{-2} \widetilde{\chi}_{\alpha \beta} \nu^{\alpha} \wedge \nu \beta
$$

and the field strength $\hat{G}_{(3)}=d \hat{B}_{(2)}$ is given by

$\hat{G}_{(3)}=G_{(3)}+g^{-1}\left(\widetilde{G}_{(2) \alpha}-m g^{-1} F_{\alpha}\right) \wedge \nu^{\alpha}+\frac{1}{2} g^{-2} G_{(1) \alpha \beta} \wedge \nu^{\alpha} \wedge \nu^{\beta}+\frac{1}{6} g^{-3} G_{(0) \alpha \beta \gamma} \nu^{\alpha} \wedge \nu^{\beta} \wedge \nu^{\gamma}$,

where

$$
\begin{aligned}
G_{(3)} & =d \widetilde{B}_{(2)}+\widetilde{B}_{(1) \alpha} \wedge F^{\alpha}-m g^{-1} \omega_{(3)}, \\
\widetilde{G}_{(2) \alpha} & =D \widetilde{B}_{(1) \alpha}+\widetilde{\chi}_{\alpha \beta} F^{\beta}, \\
G_{(1) \alpha \beta} & =D \widetilde{\chi}_{\alpha \beta}+g f_{\alpha \beta}^{\gamma} \widetilde{B}_{(1) \gamma}, \\
G_{(0) \alpha \beta \gamma} & =m f_{\alpha \beta \gamma}-3 g \widetilde{\chi}_{\delta[\alpha} f_{\beta \gamma]}^{\delta},
\end{aligned}
$$

and $\omega_{(3)}$ is the Yang-Mills Chern-Simons 3-form, satisfying $d \omega_{(3)}=F^{\alpha} \wedge F_{\alpha}$, and defined by

$$
\omega_{(3)} \equiv A^{\alpha} \wedge d A_{\alpha}+\frac{1}{3} g f_{\alpha \beta \gamma} A^{\alpha} \wedge A^{\beta} \wedge A^{\gamma}
$$

It should be noted that the redefinitions for $\widetilde{\chi}_{\alpha \beta}$ and $\widetilde{\chi}_{\alpha}$ in (3.20) amount to a projection of the scalars $\chi_{\alpha \beta}$, which are in the reducible antisymmetric product of two adjoint representations of $G$, as a sum of scalars $\tilde{\chi}_{\alpha}$ in the adjoint of $G$, and the remaining scalars $\tilde{\chi}_{\alpha \beta}$ that are orthogonal to the adjoint representation. Furthermore, it can be seen from (3.21) and (3.23) that the scalars $\tilde{\chi}_{\alpha}$ in the adjoint representation have disappeared entirely from the ansatz. In fact what has happened is that the vectors $B_{(1) \alpha}$, which themselves are also in the adjoint representation, have become massive by eating the scalars $\tilde{\chi}_{\alpha}$. This becomes clear if we write out the expression for the dimensional reduction of the $(n+q)$-dimensional 3-form Lagrangian $-\frac{1}{2} e^{\hat{a}} \hat{\phi} \hat{*} \hat{G}_{(3)} \wedge \hat{G}_{(3)}$, which can easily be seen from (3.22) and (3.23) to 
be given by

$$
\begin{aligned}
\mathcal{L}_{3}= & -\frac{1}{2} e^{\hat{a} \hat{\phi}-4 \alpha \varphi} * G_{(3)} \wedge G_{(3)}-\frac{1}{2} e^{\hat{a} \hat{\phi}-2(\alpha+\beta) \varphi} h^{\alpha \beta} *\left(G_{(2) \alpha}-\frac{m}{g} F_{\alpha}\right) \wedge\left(G_{(2) \beta}-\frac{m}{g} F_{\beta}\right) \\
& -\frac{1}{2} e^{\hat{a} \hat{\phi}-4 \beta \varphi} h^{\alpha \beta} h^{\gamma \delta} * D \widetilde{\chi}_{\alpha \gamma} \wedge D \widetilde{\chi}_{\beta \delta}-\frac{1}{2} g^{2} e^{\hat{a} \hat{\phi}-4 \beta \varphi} h^{\alpha \beta} h^{\gamma \delta} f_{\alpha \gamma}^{\lambda} f_{\beta \delta}^{\sigma} * \widetilde{B}_{(1) \lambda} \wedge \widetilde{B}_{(1) \sigma} \\
& -\frac{1}{12} m^{2} e^{\hat{a} \hat{\phi}+2(\alpha-3 \beta) \varphi} h^{\alpha_{1} \beta_{1}} h^{\alpha_{2} \beta_{2}} h^{\alpha_{3} \beta_{3}} f_{\alpha_{1} \alpha_{2} \alpha_{3}} f_{\beta_{1} \beta_{2} \beta_{3}} * \mathbb{1} \\
& -\frac{1}{4} g^{2} e^{\hat{a} \hat{\phi}+2(\alpha-3 \beta) \varphi} h^{\alpha_{1} \beta_{1}} h^{\alpha_{2} \beta_{2}} h^{\alpha_{3} \beta_{3}} f_{\alpha_{2} \alpha_{3}}^{\delta} \widetilde{\chi}_{\delta \alpha_{1}}\left(\widetilde{\chi}_{\lambda \beta_{1}} f_{\beta_{2} \beta_{3}}^{\lambda}+2 \widetilde{\chi}_{\lambda \beta_{3}} f_{\beta_{1} \beta_{2}}^{\lambda}\right) * \mathbb{1} .
\end{aligned}
$$

We can also see from this Lagrangian that not only do the vectors $\widetilde{B}_{(1) \alpha}$ have masses proportional to the gauge coupling constant $g$, but so do the remaining uneaten scalar fields $\widetilde{\chi}_{\alpha \beta}$.

It should be noted that if we consider the special case where the group manifold is $G=S U(2)$, then $\widetilde{\chi}_{\alpha \beta}$ is identically zero, since the antisymmetric product of two adjoint representations of $S U(2)$ yields only the adjoint representation. Thus in this special case, there are no scalars at all coming from the reduction of $\hat{B}_{(2)}$, since they are all eaten by the vectors $B_{(1) \alpha}$.

\subsection{The Lagrangian and gauge symmetries}

Having obtained the DeWitt reduction of the metric and the 3-form in the previous two subsections, we now put these results together to give the complete result for the DeWitt reduction of the bosonic string.

It is convenient at this stage to perform a redefinition of the scalars $\hat{\phi}$ and $\varphi$ (i.e. the $(n+q)$-dimensional dilaton and the breathing mode from the reduction), amounting to an $S O(2)$ rotation. Specifically, we rotate these two fields $(\hat{\phi}, \varphi)$ to $(\phi, \tilde{\varphi})$, where $\phi$ is taken to be proportional to the combination $\hat{a} \hat{\phi}-4 \alpha \varphi$ that appears in the exponential prefactor of the 3 -form kinetic term in (3.25). In other words, the new scalar $\phi$ can be viewed as the "dilaton" in the reduced $n$-dimensional theory. We therefore define

$$
\phi=\frac{\hat{a}}{a} \hat{\phi}-\frac{4 \alpha}{a} \varphi, \quad \tilde{\varphi}=\frac{\hat{a}}{a} \varphi+\frac{4 \alpha}{a} \hat{\phi}
$$

where

$$
a^{2}=\frac{8}{n-2}
$$

The inverse relation is

$$
\hat{\phi}=\frac{\hat{a}}{a} \phi+\frac{4 \alpha}{a} \tilde{\varphi}, \quad \varphi=\frac{\hat{a}}{a} \tilde{\varphi}-\frac{4 \alpha}{a} \phi
$$


In terms of the redefined fields, the total $n$-dimensional Lagrangian, obtained by summing $\mathcal{L}_{\mathrm{EH}}$ given in $(3.15), \mathcal{L}_{3}$ given in (3.25), and the kinetic term for $\hat{\phi}$, is

$$
\begin{aligned}
\mathcal{L}= & R * \mathbb{1}-\frac{1}{2} * d \phi \wedge d \phi-\frac{1}{2} * d \tilde{\varphi} \wedge d \tilde{\varphi}-* P_{i j} \wedge P_{i j}-\frac{1}{2} e^{\frac{1}{2} a \phi-\gamma \tilde{\varphi}} h_{\alpha \beta} * F^{\alpha} \wedge F^{\beta} \\
& -\frac{1}{2} e^{a \phi} * G_{(3)} \wedge G_{(3)}-\frac{1}{2} e^{\frac{1}{2} a \phi-\gamma \tilde{\varphi}} h^{\alpha \beta} *\left(G_{(2) \alpha}-m g^{-1} F_{\alpha}\right) \wedge\left(G_{(2) \beta}-m g^{-1} F_{\beta}\right) \\
& -\frac{1}{2} e^{-2 \gamma \tilde{\varphi}} h^{\alpha \beta} h^{\gamma \delta} * D \tilde{\chi}_{\alpha \gamma} \wedge D \tilde{\chi}_{\beta \delta}-\frac{1}{2} g^{2} e^{-2 \gamma \tilde{\varphi}} h^{\alpha \beta} h^{\gamma \delta} f_{\alpha \gamma}^{\lambda} f_{\beta \delta}^{\sigma} * \widetilde{B}_{(1) \lambda} \wedge \widetilde{B}_{(1) \sigma} \\
& -V * \mathbb{1}
\end{aligned}
$$

where we have defined

$$
\gamma \equiv \sqrt{\frac{2}{q}}
$$

and the potential $V$ for the scalar fields is given by

$$
\begin{aligned}
V= & \frac{1}{4} g^{2} e^{-\frac{1}{2} a \phi-\gamma \tilde{\varphi}}\left(h_{\alpha \beta} h^{\gamma \delta} h^{\rho \sigma} f_{\gamma \rho}^{\alpha} f_{\delta \sigma}^{\beta}+2 h^{\alpha \beta} f_{\delta \alpha}^{\gamma} f_{\gamma \beta}^{\delta}\right) \\
& +\frac{1}{12} m^{2} e^{-\frac{1}{2} a \phi-3 \gamma \tilde{\varphi}} h^{\alpha_{1} \beta_{1}} h^{\alpha_{2} \beta_{2}} h^{\alpha_{3} \beta_{3}} f_{\alpha_{1} \alpha_{2} \alpha_{3}} f_{\beta_{1} \beta_{2} \beta_{3}} \\
& +\frac{1}{4} g^{2} e^{-\frac{1}{2} a \phi-3 \gamma \tilde{\varphi}} h^{\alpha_{1} \beta_{1}} h^{\alpha_{2} \beta_{2}} h^{\alpha_{3} \beta_{3}} f_{\alpha_{2} \alpha_{3}}^{\delta} \tilde{\chi}_{\delta \alpha_{1}}\left(\widetilde{\chi}_{\lambda \beta_{1}} f_{\beta_{2} \beta_{3}}+2 \widetilde{\chi}_{\lambda \beta_{3}} f_{\beta_{1} \beta_{2}}\right),
\end{aligned}
$$

The Lagrangian (3.29) is invariant under $G$ gauge transformations of the Yang-Mills potentials $A_{(1)}^{\alpha}$, with all the other fields transforming homogeneously, according to their YangMills index structure. Note, in particular, that the 1-forms $\widetilde{B}_{(1) \alpha}$ transform homogeneously in the adjoint representation of $G$. This should be contrasted with the original 1-forms $B_{(1) \alpha}$ appearing in the reduction ansatz (3.16), which do not transform covariantly. Indeed, this is one reason why it was advantageous to make the redefinition to $\widetilde{B}_{(1) \alpha}$ in (3.20). The scalar $\phi$ deserves its appellation "dilaton," since under $g_{\mu \nu} \longrightarrow \lambda^{2} g_{\mu \nu}, \phi \longrightarrow \phi+4 a^{-1} \log \lambda$ the Lagrangian (3.29) scales uniformly as $\lambda^{n-2}$.

\section{Consistent Truncation of the Scalar Moduli}

A careful inspection of the equations of motion that result from the reduced Lagrangian (3.29) reveals that a consistent truncation is possible in which the non-singlet scalars in the metric ansatz (3.3) are set to zero, along with $\tilde{\varphi}$ and the scalars $\tilde{\chi}_{\alpha \beta}$ in the $\hat{B}_{(2)}$ ansatz (3.21), provided that at the same time we set the gauge bosons from the metric reduction and the vectors $\widetilde{B}_{(1) \alpha}$ in the $\hat{B}_{(2)}$ ansatz equal:

$$
h_{\alpha \beta}=\delta_{\alpha \beta}, \quad \tilde{\varphi}=0, \quad \tilde{\chi}_{\alpha \beta}=0, \quad \widetilde{B}_{(1) \alpha}=A_{\alpha} .
$$


We must also set the gauge-coupling constant and the 3 -form flux parameter equal ${ }^{6}$

$$
g=m \text {. }
$$

The reduction ansatz is now given simply by

$$
\begin{aligned}
d \hat{s}^{2} & =e^{a q /(2(n+q-2)) \phi} d s_{n}^{2}+m^{-2} e^{-a(n-2) /(2(n+q-2)) \phi} g_{\alpha \beta} \nu^{\alpha} \nu^{\beta}, \\
\hat{B}_{(2)} & =m^{-2} \tilde{\omega}_{(2)}+\widetilde{B}_{(2)}+m^{-1} A_{\alpha} \wedge \nu^{\alpha}, \\
\hat{\phi} & =\sqrt{\frac{n-2}{n+q-2}} \phi .
\end{aligned}
$$

The fact that this rather remarkable consistent truncation is possible in any group manifold reduction of the bosonic string was first discovered in [11], where the same ansatz as (4.3) was presented (in the string frame rather than the Einstein frame we are using here). The lower-dimensional Lagrangian that describes the truncated theory is simply obtained by imposing the relations (4.1) in (3.29):

$$
\mathcal{L}=R * \mathbb{1}-\frac{1}{2} * d \phi \wedge d \phi-\frac{1}{2} e^{a \phi} * G_{(3)} \wedge G_{(3)}-\frac{1}{2} e^{\frac{1}{2} a \phi} * F^{\alpha} \wedge F^{\alpha}+\frac{1}{3} q m^{2} e^{-\frac{1}{2} a \phi} * \mathbb{1} .
$$

In [11], the higher-dimensional bosonic string theory was augmented by the inclusion of the conformal anomaly term

$$
\mathcal{L}_{\text {conf }}=-k^{2}(n+q-26) e^{-\frac{1}{2} \hat{\alpha} \hat{\phi}}
$$

in (2.10), and it was shown that with the same reduction ansatz (4.3) this additional term can cancel the scalar potential in (4.4), implying that one obtains precisely the bosonic sector of the effective action for the heterotic string.

In section 7 a consistent truncation of the Lagrangian 3.29 with two singlet scalars $\Phi$ and $\tilde{\phi}$ is given. It would be interesting to address other possible consistent truncations with more scalars turned on and address possible domain wall solutions there.

\section{$5 \quad$ DeWitt $=$ Pauli $\circ$ Kaluza}

In section 3, we described the DeWitt reduction of the bosonic string on a group manifold $G$, in which one keeps all the fields that are singlets under the left action of $G$. This reduction, as with all DeWitt reductions, is guaranteed to be consistent, by virtue of the left-acting group invariance of the ansatz. By contrast, if one attempts a generalisation of

\footnotetext{
${ }^{6}$ To be precise, we can leave $g$ and $m$ unequal, and instead set $\tilde{\varphi}=\tilde{\varphi}_{0}$ where $g=m e^{-2 \gamma \tau \varphi_{0}}$, but for simplicity, and without loss of generality, we can choose the constant $\tilde{\varphi}_{0}$ to be zero and hence $g=m$.
} 

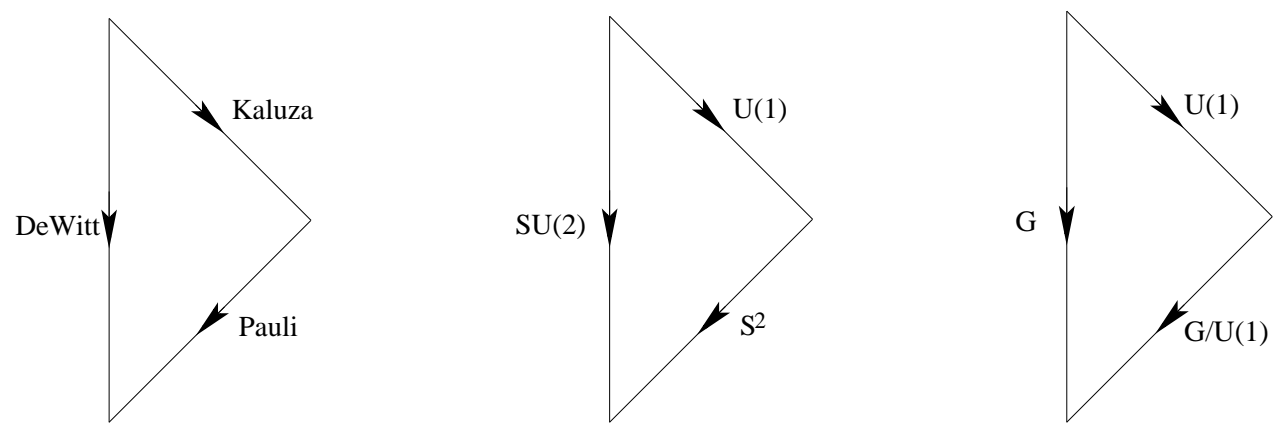

Figure 1: DeWitt reduction on a group manifold $G$, composed as a Kaluza reduction on $U(1)$ followed by a Pauli reduction on $G / U(1)$.

the reduction idea to a case where the internal manifold is a coset space, such as a sphere, then aside from exceptional cases it is not possible to perform a consistent reduction that retains a finite set of lower-dimensional fields including all the gauge bosons of the isometry group. Furthermore, in those exceptional cases where such a consistent reduction is possible, there is currently no clear understanding, for example from group theory, as to why the consistency is achieved.

In this section we shall explore some features of coset reductions, which we refer to as "Pauli reductions" since the original such example, of a 2 -sphere reduction from six dimensions, was considered by Pauli in 1953 [5] (see [6, 7]). We shall describe the construction of certain classes of theory that do admit consistent Pauli coset reductions on $G / H$. Namely, any theory that can itself be obtained from a yet higher dimensional "progenitor" theory as a DeWitt group manifold reduction on $H$ admits a consistent Pauli reduction on $G / H$, for any group $G$ that contains $H$. The theory that results from the Pauli reduction on $G / H$ will be the same as the theory obtained by making a DeWitt reduction of the progenitor theory on $G$. The metrics induced on $G / H$ in this description are precisely those for the consistent Pauli reduction, including the gauge bosons of the group $G$ of isometries of $G / H$.

We shall demonstrate the above procedure in a variety of cases; first for $S U(2) / U(1)$, then $G / U(1)$ for arbitrary $G$, and finally $G / H$ for any reductive coset. The first two, which are examples of the DeWitt $=$ Pauli $\circ$ Kaluza composition, are illustrated in Figure 1 below . The $G / H$ case, which is a DeWitt $=$ Pauli $\circ$ DeWitt composition, is illustrated in Figure 2 . 

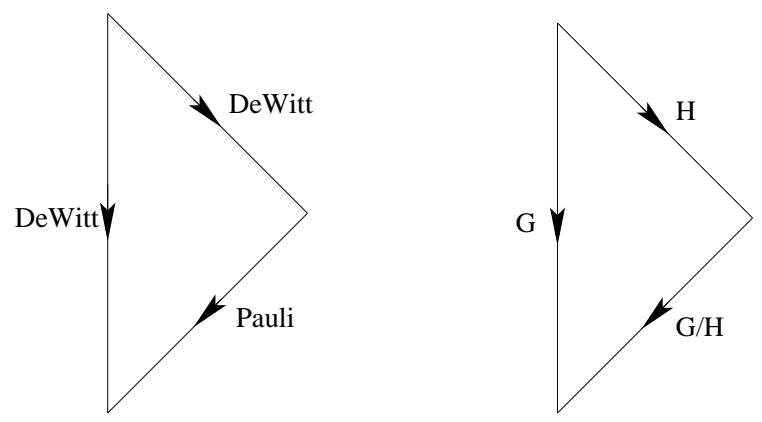

Figure 2: DeWitt reduction on $G$, composed as a DeWitt reduction on $H$ followed by a Pauli reduction on $G / H$.

\subsection{The case $S U(2) / U(1)$}

In this section, we consider the case of the DeWitt reduction on $S^{3}$ of pure Einstein gravity, showing how, by instead first reducing the Einstein theory on $S^{1}$, we can obtain a consistent Pauli reduction of an Einstein-Maxwell-dilaton system on $S^{2}$. This makes contact with a result in [24], where it was shown that if one starts in $D$ dimensions with the theory described by the Lagrangian

$$
\mathcal{L}_{D}=R * \mathbb{1}-\frac{1}{2} * d \phi \wedge d \phi-\frac{1}{2} e^{-a \phi} * F_{(2)} \wedge F_{2},
$$

where the dilaton coupling constant $a$ is given by

$$
a^{2}=\frac{2(D-1)}{D-2}
$$

then one can perform a consistent $S^{2}$ reduction that includes, in particular, the $S U(2)$ gauge bosons associated with the isometry group of the sphere. This specific value of the dilaton coupling is precisely the one that arises if $(D+1)$-dimensional pure gravity is reduced on $S^{1}$ (a Kaluza reduction). Thus the consistent $S^{2}$ reduction of (5.1) derived in [24] can be interpreted as a consistent reduction of $(D+1)$-dimensional gravity where there is a first step of reduction on $S^{1}$ followed by the reduction on $S^{2}$. As we shall show, the $S^{1}$ fibre has a non-trivial twist, and the whole reduction can be reinterpreted as a DeWitt reduction from $(D+1)$ dimensions on the group manifold $S U(2) \sim S^{3}$.

We begin by writing down the standard DeWitt reduction of $(D+1)$-dimensional pure Einstein gravity on the group manifold $S U(2)$; the metric ansatz is given by

$$
d s_{D+1}^{2}=e^{2 \alpha \varphi} d s_{D-2}^{2}+e^{2 \beta \varphi} \widetilde{T}_{i j}\left(\sigma^{i}-A^{i}\right)\left(\sigma^{j}-A^{j}\right),
$$

where $d \sigma^{i}=-\frac{1}{2} \epsilon_{i j k} \sigma^{j} \wedge \sigma^{k}$, and $\widetilde{T}_{i j}$ denotes the unimodular matrix of scalar fields. The 
$S U(2)$ left-invariant 1-forms can be written in terms of Euler angles $(\theta, \tau, \psi)$ as $^{7}$

$$
\sigma_{1}=\cos \psi d \theta+\sin \psi \sin \theta d \tau, \quad \sigma_{2}=-\sin \psi d \theta+\cos \psi \sin \theta d \tau, \quad \sigma_{3}=d \psi+\cos \theta d \tau
$$

The constants $\alpha$ and $\beta$ in (5.3) are given by

$$
\alpha^{2}=\frac{3}{2(D-4)(D-1)}, \quad \beta=-\frac{\alpha(D-4)}{3} .
$$

The next step is to introduce Cartesian coordinates $\mu^{i}$ on $\mathbb{R}^{3}$, subject to the constraint $\mu^{i} \mu^{i}=1$, which defines the unit $S^{2}$. We relate the $\mu^{i}$ to the previously-introduced Euler angles by setting

$$
\mu_{1}=\sin \psi \sin \theta, \quad \mu_{2}=\cos \psi \sin \theta, \quad \mu_{3}=\cos \theta
$$

A straightforward calculation shows that we shall have

$$
\sigma^{i}-A^{i}=-\epsilon_{i j k} \mu^{j} \mathcal{D} \mu^{k}+\mu^{i} \sigma
$$

where

$$
\sigma \equiv d \tau+\cos \theta d \psi-\mu^{i} A^{i}, \quad \mathcal{D} \mu^{i} \equiv d \mu^{i}+\epsilon_{i j k} A^{j} \mu^{k} .
$$

After some involved but mechanical manipulations, we find that we can write (5.3) as

$$
d \hat{s}_{D+1}^{2}=e^{2 \alpha \varphi} d s_{D-2}^{2}+e^{2 \beta \varphi} \widetilde{\Delta}^{-1} \widetilde{T}_{i j}^{-1} \mathcal{D} \mu^{i} \mathcal{D} \mu^{j}+e^{2 \beta \varphi} \Delta(d \tau+A)^{2},
$$

where

$$
\begin{aligned}
A & =\cos \theta d \psi-\mu^{i} A^{i}-\widetilde{\Delta}^{-1} \widetilde{T}_{i j} \epsilon_{i k \ell} \mu^{j} \mu^{k} \mathcal{D} \mu^{\ell}, \\
\widetilde{\Delta} & \equiv \widetilde{T}_{i j} \mu^{i} \mu^{j}
\end{aligned}
$$

Since $\tau$ is an isometry direction, we can now perform a standard Kaluza reduction of (5.9) on the circle parameterised by $\tau$, by writing it as

$$
d \hat{s}_{D+1}^{2}=e^{2 \tilde{\alpha} \phi} d s_{D}^{2}+g^{-2} e^{2 \tilde{\beta} \phi}(d \tau+A)^{2},
$$

where

$$
\tilde{\alpha}^{2}=\frac{1}{2(D-1)(D-2)}, \quad \tilde{\beta}=-(D-2) \tilde{\alpha} .
$$

Since we are starting from pure Einstein gravity in $(D+1)$ dimensions, it follows that the metric $d s_{D}^{2}$, the vector potential $A$ and the dilaton $\phi$ will satisfy the equations of motion for the $D$-dimensional Einstein-Maxwell-dilaton system described by the Lagrangian (5.1).

\footnotetext{
${ }^{7}$ We are using $\tau$ rather than the more usual $\phi$ for one of the Euler angles here since $\phi$ is already in use as a dilaton.
} 
Comparing with (5.9), we see that this gives the following $S^{2}$ reduction ansatz from $D$ dimensions to $(D-2)$ dimensions:

$$
\begin{aligned}
d s_{D}^{2} & =e^{2 \alpha \varphi-2 \tilde{\alpha} \phi} d s_{D-2}^{2}+g^{-2} e^{2 \beta \varphi-2 \tilde{\alpha} \phi} \widetilde{\Delta}^{-1} \widetilde{T}_{i j}^{-1} D \mu^{i} D \mu^{j} \\
A & =\cos \theta d \psi-\mu^{i} A^{i}-\widetilde{\Delta}^{-1} \widetilde{T}_{i j} \epsilon_{i k \ell} \mu^{j} \mu^{k} D \mu^{\ell} \\
e^{2 \tilde{\beta} \phi} & =e^{2 \beta \varphi} \widetilde{\Delta}
\end{aligned}
$$

It is straightforward to re-express this reduction ansatz as

$$
\begin{aligned}
d s_{D}^{2} & =Y^{\frac{1}{D-2}}\left(\Delta^{\frac{1}{D-2}} d s_{D-2}^{2}+g^{-2} \Delta^{-\frac{D-3}{D-2}} T_{i j}^{-1} D \mu^{i} D \mu^{j}\right), \\
e^{\sqrt{\frac{2(D-2)}{D-1} \phi}} & =\Delta^{-1} Y^{\frac{D-3}{D-1}} \\
A & =\cos \theta d \psi-\mu^{i} A^{i}-\Delta^{-1} T_{i j} \epsilon_{i k \ell} \mu^{j} \mu^{k} D \mu^{\ell},
\end{aligned}
$$

where we have absorbed the breathing-mode scalar $\varphi$ introduced in (5.3) into the matrix $T_{i j}$, defined in terms of the unimodular matrix $\widetilde{T}_{i j}$ by $T_{i j} \equiv Y^{1 / 3} \widetilde{T}_{i j}$, and $Y$ is given by $Y=e^{(D-1) \alpha \varphi}$. After a somewhat lengthy calculation, we find that the field strength $F=d A$ is then expressible as

$$
\begin{aligned}
F= & \frac{1}{2} \epsilon_{i j k}\left(g^{-1} U \Delta^{-2} \mu^{i} D \mu^{j} \wedge D \mu^{k}-2 g^{-1} \Delta^{-2} D \mu^{i} \wedge D T_{j \ell} T_{k m} \mu^{\ell} \mu^{m}\right) \\
& -\Delta^{-1} T_{i j} \mu^{i} F^{j} .
\end{aligned}
$$

The $S^{2}$ reduction that we have derived here is precisely the consistent Pauli reduction ansatz derived first in [24].

It should be emphasised that although we have focused here on the $S^{3}$ DeWitt reduction of pure Einstein gravity in $(D+1)$ dimensions, and its consequent reinterpretation as a Pauli reduction of the $D$-dimensional Einstein-Maxwell-dilaton system, we could just as well begin with any theory in $(D+1)$ dimensions. That theory, reduced on a circle, will then, by the same arguments, yield a $D$-dimensional theory that can necessarily be consistently Pauli-reduced on $S^{2}$. Likewise, the results in the next subsection extend to show that this $D$-dimensional theory can be consistently Pauli-reduced on any coset $G / U(1)$.

An example of a $D$-dimensional theory that can be obtained from a circle reduction is type IIA supergravity, since it comes from the $S^{1}$ reduction of eleven-dimensional supergravity. Thus, for example, we are guaranteed to be able to find a consistent Pauli reduction of type IIA supergravity on $S^{2}$. In fact the resulting eight-dimensional theory will be precisely the same $S U(2)$-gauged supergravity as the one obtained in [27] by performing the DeWitt reduction of eleven-dimensional supergravity on $S^{3}=S U(2)$. 


\subsection{The case $G / U(1)$}

In this section, we show how the previous discussion can be generalised to the case of any group $G$ factored by $U(1)$. We begin by introducing generators $T_{a}$ for the Lie algebra of $G$, and defining the left-invariant and right-invariant 1-forms of $G$ by

$$
\lambda^{a} T_{a}=V^{-1} d V, \quad \rho^{a} T_{a}=d V V^{-1},
$$

where $V \in G$. It is evident that the matrix $U^{a}{ }_{b}$ defined by

$$
T_{a} U_{b}^{a}=V^{-1} T_{b} V
$$

has the properties

$$
U^{a}{ }_{c} U_{b}{ }^{c}=U^{c}{ }_{b} U_{c}^{a}=\delta_{b}^{a},
$$

where indices are raised and lowered using the Cartan-Killing metric $g_{a b}=-\operatorname{tr}\left(T_{a} T_{b}\right)$. We can use $U_{b}^{a}$ to relate the left-invariant and right-invariant 1-forms:

$$
\lambda^{a}=U_{b}^{a} \rho^{b}, \quad \rho^{a}=U_{b}{ }^{a} \lambda^{b} .
$$

We now split the generators as $T_{a}=\left(T_{0}, T_{i}\right)$, where $T_{0}$ is the generator of the relevant $U(1)$ subgroup, and parameterise group elements $V \in G$ in the form

$$
V=e^{\tau T_{0}} \widetilde{V}
$$

where $\widetilde{V}$ parameterises elements in the coset $G / U(1)$. Thus, in particular, $\widetilde{V}$ is independent of the coordinate $\tau$ on the $U(1)$ circle. From (5.16), we find

$$
\begin{aligned}
\rho^{a} T_{a} & =d \tau T_{0}+e^{\tau T_{0}} d \widetilde{V} \widetilde{V}^{-1} e^{-\tau T_{0}} \\
& =(d \tau+\omega) T_{0}+\rho^{i} T_{i},
\end{aligned}
$$

where $d \widetilde{V} \widetilde{V}^{-1}=\omega T_{0}+\tilde{\rho}^{i} T_{i}$ and $\rho^{i} T_{i}=e^{\tau T_{0}} T_{i} e^{-\tau T_{0}} \tilde{\rho}^{i}$. Note that $\omega$ and $\tilde{\rho}^{i}$ are independent of $\tau$.

From (5.16), the left-invariant 1-forms $\lambda^{a}$ are given by

$$
\begin{aligned}
\lambda^{a} T_{a} & =d \tau \widetilde{V}^{-1} T_{0} \widetilde{V}+\widetilde{V}^{-1} d \widetilde{V} \\
& =\left(U^{a}{ }_{0} \rho^{0}+U^{a}{ }_{i} \rho^{i}\right) T_{a},
\end{aligned}
$$

where the second line follows from (5.17). Now, it is evident by setting $b=0$ in (5.17) that we have

$$
U_{0}^{a}=V^{-1} T_{0} V=\widetilde{V}^{-1} T_{0} \tilde{V}
$$


and hence that $U^{a}{ }_{0}$ is independent of $\tau$. It is also evident that

$$
U^{a}{ }_{i} \rho^{i} T_{a}=V^{-1}\left(\rho^{i} T_{i}\right) V=\widetilde{V}^{-1} e^{-\tau T_{0}}\left(\rho^{i} T_{i}\right) e^{\tau T_{0}} \widetilde{V}=\widetilde{V}^{-1}\left(\tilde{\rho}^{i} T_{i}\right) \tilde{V},
$$

which, from the already-established $\tau$-independence of $\tilde{\rho}^{i}$ implies that $U^{a}{ }_{i} \rho^{i}$ is independent of $\tau$. The upshot of these observations is that, from (5.21) and (5.22), we have

$$
\lambda^{a}=U_{0}^{a}(d \tau+\omega)+U^{a}{ }_{i} \rho^{i},
$$

where $U^{a}{ }_{0}, \omega$ and $U^{a}{ }_{i} \rho^{i}$ are all independent of $\tau$, and of course $\rho^{i}$ is orthogonal to $\partial / \partial \tau$.

Armed with these preliminaries, we can now consider the standard DeWitt metric reduction on the group manifold $G$ :

$$
d \hat{s}^{2}=e^{2 \alpha \varphi} d s^{2}+g^{-2} e^{2 \beta \varphi} T_{a b}\left(\lambda^{a}-A^{a}\right)\left(\lambda^{b}-A^{b}\right),
$$

(It is understood here that $T_{a b}$ is taken to be unimodular; also, without loss of generality we are setting the gauge coupling constant $g=1$.) Substituting (5.25) into this, we obtain

$$
d \hat{s}^{2}=e^{2 \alpha \varphi} d s^{2}+e^{2 \beta \varphi} \Delta\left(d \tau+\omega-U_{a}^{0} A^{a}\right)^{2}+2 T_{a b} U_{0}^{a}\left(d \tau+\omega-U_{a}^{0} A^{a}\right) h^{b}+T_{a b} h^{a} h^{b},
$$

where we have defined

$$
h^{a} \equiv U_{i}^{a}\left(\rho^{i}-U_{b}^{i} A^{b}\right), \quad \Delta \equiv T_{a b} U^{a}{ }_{0} U^{b}{ }_{0} .
$$

Note that since $U^{a}{ }_{i} U_{b}{ }^{i}=U^{a}{ }_{c} U_{b}{ }^{c}-U^{a}{ }_{0} U_{b}{ }^{0}=\delta_{b}^{a}-U^{a}{ }_{0} U_{b}{ }^{0}$, and we have already established that $U^{a}{ }_{0}$ is independent of $\tau$, it follows that $U^{a}{ }_{i} U_{b}{ }^{i}$ is also independent of $\tau$, and hence so is $h^{a}$. Thus by taking all the fields $d s^{2}, T_{a b}, \varphi$ and $A^{a}$ to be independent of $\tau$, we have a $U(1)$ isometry generated by $\partial / \partial \tau$, which can be used for a standard Kaluza reduction. To do this, we complete the square in (5.27), giving

$$
\begin{aligned}
d \hat{s}^{2}= & e^{2 \alpha \varphi} d s^{2}+e^{2 \beta \varphi}\left(d \tau+\omega-U_{a}^{0} A^{a}+\Delta^{-1} T_{a b} U^{a}{ }_{0} h^{b}\right)^{2} \\
& +e^{2 \beta \varphi}\left(T_{a b}-\Delta^{-1} T_{a c} T_{b d} U^{c}{ }_{0} U^{d}{ }_{0}\right) h^{a} h^{b} .
\end{aligned}
$$

If we start from pure Einstein gravity in $(D+1)$ dimensions, and reduce on the $U(1)$ isometry generated by $\partial / \partial \tau$ using the standard reduction ansatz (5.11), we therefore find that the DeWitt reduction of the $(D+1)$-dimensional theory on $G$ can be reinterpreted as a Pauli reduction on the coset $G / U(1)$ of the $D$-dimensional Einstein-Maxwell-dilaton theory (5.1), with the reduction ansatz given by

$$
\begin{aligned}
d s_{D}^{2} & =\left(e^{2 \beta \varphi} \Delta\right)^{-\frac{1}{D-2}}\left(e^{2 \alpha \varphi} d s^{2}+e^{2 \beta \varphi}\left(T_{a b}-\Delta^{-1} T_{a c} T_{b d} U_{0}^{c} U_{0}^{d} h^{a} h^{b}\right),\right. \\
A_{(1)} & =\omega-U_{a}{ }^{0} A^{a}+\Delta^{-1} T_{a b} U^{a}{ }_{0} h^{b}, \\
e^{2 \tilde{\beta} \phi} & =e^{2 \beta \varphi} \Delta .
\end{aligned}
$$


The automatic consistency of the DeWitt reduction on $G$ ensures the consistency of this Pauli reduction on $G / U(1)$.

It is instructive to make contact with our results for the case $S U(2) / U(1)$ in section 5.1. To do this, we note that the terms in the metric reduction (5.30) in the coset directions can be written as

$$
\left(e^{2 \beta \varphi} \Delta\right)^{-\frac{1}{D-2}} e^{2 \beta \varphi} \Delta^{-1}\left(T_{a b} T_{c d}-T_{a c} T_{b d}\right) U_{0}^{c} U_{0}^{d} h^{a} h^{b}
$$

Since $T_{a b}$ is $3 \times 3$ and unimodular, we have

$$
T_{a b} T_{c d}-T_{a c} T_{b d}=\epsilon_{a c e} \epsilon_{b d f} T_{e f}^{-1}
$$

Defining $\tilde{h}^{a}=\epsilon_{a b c} h^{b} U_{0}^{c}$, we find that the metric reduction in (5.30) can be written in this special case as

$$
d s_{D}^{2}=\left(e^{2 \beta \varphi} \Delta\right)^{-\frac{1}{D-2}}\left(e^{2 \alpha \varphi} d s^{2}+e^{2 \beta \varphi} \Delta^{-1} T_{a b}^{-1} \tilde{h}^{a} \tilde{h}^{b}\right)
$$

This can be seen to be equivalent to the metric reduction in (5.13), with $U_{0}^{a}=\mu^{a}$ and $\tilde{h}^{a}=\mathcal{D} \mu^{a}$. Likewise, the reductions for $A_{(1)}$ and $\phi$ in (5.30) coincide in this case with the expressions in (5.13).

\subsection{The case $G / H$}

It should now be clear that the procedure we have described in section 5.2 for $G / U(1)$ can be generalised to any $\operatorname{coset} G / H$. This can easily seen if we parameterise the group element $V \in G$ as

$$
V=h k
$$

where $h \in H$ and $k \in G / H$. The DeWitt reduction on $G$ is written in terms of $T_{a b}\left(\lambda^{a}-\right.$ $\left.A^{a}\right)\left(\lambda^{b}-A^{b}\right)$, and so one needs to evaluate

$$
T_{a}\left(\lambda^{a}-A^{a}\right)=V^{-1} d V-A
$$

where $A \equiv T_{a} A^{a}$, and $T_{a}$ are the generators of $G$. One then has

$$
\begin{aligned}
T_{a}\left(\lambda^{a}-A^{a}\right) & =k^{-1} h^{-1} d h k+k^{-1} d k-A \\
& =k^{-1}\left(h^{-1} d h+d k k^{-1}-k A k^{-1}\right) k
\end{aligned}
$$

The terms enclosed within the parentheses in the second line of (5.36) can be split into the contribution $h^{-1} d h+\left(d k k^{-1}\right)_{\|}+\left(k A k^{-1}\right)_{\|}$parallel to the $h$ fibres, and the contribution $\left(d k k^{-1}\right)_{\perp}+\left(k A k^{-1}\right)_{\perp}$ perpendicular to the fibres (i.e. in the $G / H$ base). 
We split the generators $T_{a}$ of $G$ as $T_{a}=\left(T_{\alpha}, T_{i}\right)$, where $T_{\alpha}$ generate the subgroup $H$. One can parameterise elements in $H$ as

$$
h=e^{\tau_{\alpha} T_{\alpha}} .
$$

The left-invariant 1-forms $\lambda^{a}$ for $G$ are then given by

$$
\lambda^{a} T_{a}=\Lambda^{\alpha} k^{-1} T_{\alpha} k+k^{-1} d k,
$$

where $\Lambda^{\alpha}$ are the left-invariant 1-forms on the subgroup $H$ :

$$
\Lambda^{\alpha} T_{\alpha}=h^{-1} d h
$$

It is clear from (5.38) that one can substitute the $\lambda^{a}$ into (5.26), and then perform a DeWitt reduction on the fibres of the group manifold $H$, by completing the square on the terms involving $\Lambda^{\alpha}$. Thus one has a "DeWitt = Pauli $\circ$ DeWitt" interpretation, for any group $G$ with subgroup $H$.

If the coset is reductive, meaning in particular that $[H, K]=K$, we can obtain rather elegant explicit formulae for the reduction, which give a natural generalisation of the expressions in section 5.2. ${ }^{8}$ We derive these formulae in Appendix A.

\section{Pauli Reductions of the Bosonic String}

\subsection{Introduction}

In section 3, we discussed the standard DeWitt procedure for performing a group-manifold dimensional reduction, applied to the specific case of the $D$-dimensional bosonic string. As always in the DeWitt reduction, the consistency of the procedure is guaranteed by virtue of the fact that the ansatz is invariant under the transitively acting left action of the group $G$ on the reduction manifold. In section 4 , we discussed the details of the consistent truncation of this group manifold reduction that can be performed in the special case of the bosonic string. Namely, one can consistently set to zero all the scalar modulus fields, provided that at the same time one equates the vectors coming from the reduction of the 2 -form potential and the vectors coming from the reduction of the metric. This consistent truncation, which was first discovered in [11], results in a lower-dimensional theory comprising just gravity, the gauge bosons of $G$, and the dilaton. The fact that this truncation can be performed consistently depends on specific features of the bosonic string, and there is no obvious

\footnotetext{
${ }^{8}$ In practice the cosets occurring in dimensional reductions on compact spaces are always reductive.
} 
group-theoretic explanation for it. The calculations are sufficiently straightforward in this case, however, that one may regard the explicit demonstration of the consistency as an adequate, if unilluminating, explanation in its own right.

A more subtle situation arises if we consider more general possibilities for dimensional reduction of the bosonic string on a group manifold. Since the bi-invariant metric on the manifold $G$ has isometry group $G_{L} \times G_{R}$, one can enquire whether it is possible to perform a consistent reduction in which the gauge bosons of the entire $G_{L} \times G_{R}$ isometry group are retained. ${ }^{9}$ If one were considering such a possibility in the reduction of a generic higherdimensional theory, the answer would certainly be "no." However, the special features of the bosonic string that have already been seen to play a role in section 4 suggest that further remarkable consistent truncations may be possible. Indeed, persuasive evidence was obtained in [25] which implies that a consistent reduction of the bosonic string that retains the gauge bosons of $G \times G$ should be possible. Specifically, by considering a reduction ansatz that was exact in the gauge-boson sector, and correct up to linear order in scalar fields, it was shown that a highly non-trivial potential obstacle to the consistency of the reduction was avoided, as a consequence of certain conspiracies between contributions from the metric and the 2-form reduction ansätze. As a result, it was conjectured in [25] that there always exists a consistent reduction of the bosonic string on a group manifold $G$, in which the lower-dimensional fields comprise the metric, the dilaton, the gauge bosons of $G_{L} \times G_{R}$, and scalar fields in the representation $\left(\operatorname{Adjoint}\left(G_{L}\right)\right.$, Adjoint $\left.\left(G_{R}\right)\right)$. In the terminology of the present paper we refer to this as a Pauli reduction, since in the spirit of Pauli's proposed $S^{2}$ reduction scheme it is a case where gauge bosons for the entire isometry group of the reduction manifold are obtained.

Further supporting evidence for the consistency of this Pauli reduction comes from considerations discussed in [24]. In that paper, a general argument that yields a necessary condition for the existence of a consistent reduction was introduced. The argument is as follows. Suppose a $D$-dimensional theory is such that when dimensionally reduced on the $q$-torus it yields a theory in $(D-q)$ dimensions with a global symmetry group $P$, whose maximal compact subgroup is $Q$. If instead the $D$-dimensional theory is reduced on a compact manifold $M_{q}$, then a necessary condition for there to exist a consistent such reduction is that the gauge bosons in the reduction ansatz, coming from the isometry group of $M_{q}$, must be contained within the maximal compact subgroup $Q$ of the toroidal reduction.

\footnotetext{
${ }^{9}$ As usual, we are addressing ourselves to situations where only a finite total number of lower-dimensional fields are to be retained in the reduction ansatz.
} 
The argument for this is that one can always take a limit where the scale-size of $M_{q}$ is sent to infinity in the putative consistent reduction on $M_{q}$, and in this limit the curved manifold $M_{q}$ effectively approaches the flat torus $T^{q}$. Conversely, the lower-dimensional theory that one would obtain from the $M_{q}$ reduction can be viewed as a gauging of the theory coming from the $T^{q}$ reduction. However, the process of gauging requires that one gauge a subgroup of the maximal compact subgroup $Q$ of the global symmetry of the ungauged toroidallyreduced theory. Thus it follows that one cannot obtain a theory from the $M_{q}$ reduction whose gauge group lies outside the maximal compact subgroup $Q$.

If we now apply this argument to the case of the bosonic string, we know that after a Kaluza-type dimensional reduction on $T^{q}$ we obtain a theory with scalars in the coset $O(q, q) /(O(q) \times O(q))$, and thus the maximal compact subgroup $Q$ is $O(q) \times O(q)$. If we now instead consider reducing the bosonic string on a group manifold $G$ whose dimension is $\operatorname{dim}(G)=q$, then we see that the necessary condition for the consistency of a Pauli reduction retaining the gauge bosons of $G \times G$ is that we should have

$$
G \times G \subset O(q) \times O(q)
$$

This is in fact the case for any group $G$, since, as is well known, there is a natural embedding of any compact group $G$ in the orthogonal group $O(q)$ where $q$ is the dimension of $G$. Thus the necessary condition for the existence of a consistent Pauli reduction of the bosonic string is satisfied for any group manifold $G$.

One can easily see that special properties of the bosonic string are playing a crucial role here. If we were instead to entertain the possibility of a consistent Pauli reduction of a generic theory such as $D$-dimensional pure gravity, the analogous test would be to see whether $G \times G$ is contained in the denominator group of the $P / Q=S L(q, \mathbb{R}) / O(q)$ or $P / Q=G L(q, \mathbb{R}) / O(q)$ scalar coset that would arise in such cases. Clearly the answer is "no," and so one could not hope to retain more than the single copy of $G$ that arises in a DeWitt reduction.

\subsection{Pauli reductions of the bosonic string on $S^{3}$ and group manifolds}

In fact an example of a consistent Pauli reduction of the bosonic string on a group manifold $G$ is already known in the literature, for the case of $G=S U(2) \sim S^{3}$ [24]. A complete and explicit reduction ansatz for this example was obtained in [24], which yields a reduced theory that includes all six gauge bosons of the $S O(4)$ isometry group of the round 3-sphere, together with ten scalar fields. The reduction ansatz was given in the Einstein frame in 
[24], but for our present purposes it is simpler to work in the string frame. After making the appropriate redefinitions, the ansatz becomes

$$
\begin{aligned}
d \hat{s}_{D}^{2}= & d s_{D-3}^{2}+g^{-2} \Delta^{-1} Y^{1 / 2} T_{i j}^{-1} \mathcal{D} \mu^{i} \mathcal{D} \mu^{j}, \\
e^{-2 \hat{\phi} / \hat{a}}= & \Delta^{-1} Y^{(D-4) / 4}, \\
\hat{F}_{(3)}= & F_{(3)}+\frac{1}{6} \epsilon_{i_{1} i_{2} i_{3} i_{4}}\left(g^{-2} U \Delta^{-2} \mathcal{D} \mu^{i_{1}} \wedge \mathcal{D} \mu^{i_{2}} \wedge \mathcal{D} \mu^{i_{3}} \mu^{i_{4}}\right. \\
& \left.-3 g^{-2} \Delta^{-2} \mathcal{D} \mu^{i_{1}} \wedge \mathcal{D} \mu^{i_{2}} \wedge \mathcal{D} T_{i_{3} j} T_{i_{4} k} \mu^{j} \mu^{k}-3 g^{-1} \Delta^{-1} F_{(2)}^{i_{1} i_{2}} \wedge \mathcal{D} \mu^{i_{3}} T_{i_{4 j}} \mu^{j}\right),
\end{aligned}
$$

where

$$
\mu^{i} \mu^{i}=1, \quad \Delta=T_{i j} \mu^{i} \mu^{j}, \quad U=2 T_{i k} T_{j k} \mu^{i} \mu^{j}-\Delta T_{i i},
$$

the indices $i, j, \ldots$ range over 4 values, which we take to be $(0,1,2,3)$, and we define $Y \equiv$ $\operatorname{det}\left(T_{i j}\right)$. The gauge-covariant exterior derivative $\mathcal{D}$ is defined so that

$$
\mathcal{D} \mu^{i}=d \mu^{i}+g A_{(1)}^{i j} \mu^{j}, \quad \mathcal{D} T_{i j}=d T_{i j}+g A_{(1)}^{i k} T_{k j}+g A_{(1)}^{j k} T_{i k},
$$

where $A_{(1)}^{i j}$ denotes the $S O(4)$ gauge potentials coming from the isometry group of the 3 -sphere, and

$$
F_{(2)}^{i j}=d A_{(1)}^{i j}+g A_{(1)}^{i k} \wedge A_{(1)}^{k j}
$$

Note that $Y$ parameterises the lower-dimensional dilaton $\phi$, namely $Y=e^{-a \phi}$ with $a^{2}=$ $8 /(D-5)$. The lower-dimensional metric appearing in $(6.2)$ is written in its string frame; this is related to the Einstein frame by

$$
d s_{D-3}^{2}(\text { string })=Y^{1 / 2} d s_{D-3}^{2}(\text { Einstein })=e^{-\frac{1}{2} a \phi} d s_{D-3}^{2}(\text { Einstein }) .
$$

We would like to generalise the above $S^{3}$ Pauli reduction results to obtain an ansatz for the Pauli reduction of the bosonic string on an arbitrary group manifold. For the reduction of the field strength $\hat{F}_{(3)}$, this is a complicated problem that we have not yet succeeded in solving. For the metric reduction ansatz, on the other hand, there does seem to be a natural conjecture for its form. We can see this by first noting that in the case of $S^{3}$, the metric reduction ansatz in (6.2) can be written as

$$
d \hat{s}_{D}^{2}=d s_{D-3}^{2}+g^{-2} \widetilde{\Delta}^{-1} \widetilde{T}_{i j}^{-1} \mathcal{D} \mu^{i} \mathcal{D} \mu^{j},
$$

where $\widetilde{T}_{i j} \equiv Y^{-1 / 4} T_{i j}$ is unimodular, and $\widetilde{\Delta} \equiv \widetilde{T}_{i j} \mu^{i} \mu^{j}$. After some algebra, we find that the metric (6.7) has the inverse $(\partial / \partial \hat{s})^{2} \equiv \hat{g}^{M N} \partial_{M} \otimes \partial_{N}$, with

$$
\left(\frac{\partial}{\partial \hat{s}}\right)^{2}=\frac{1}{2} g^{2} \widetilde{T}_{i k} \widetilde{T}_{j \ell} K^{i j} \otimes K^{k \ell}+g^{\mu \nu}\left(\partial_{\mu}+\frac{1}{2} g A_{\mu}^{i j} K^{i j}\right) \otimes\left(\partial_{\nu}+\frac{1}{2} g A_{\nu}^{k \ell} K^{k \ell}\right),
$$


where $g^{\mu \nu}$ is the inverse of the lower-dimensional spacetime metric, and $K^{i j}$ denotes the $S O(4)$ Killing vectors,

$$
K^{i j}=\mu^{i} \frac{\partial}{\partial \mu_{j}}-\mu^{j} \frac{\partial}{\partial \mu^{i}} .
$$

In fact (6.8), which can be proved relatively easily by diagonalising $\widetilde{T}_{i j}$ at a point, admits an immediate generalisation to any sphere $S^{n}$ for arbitrary $n$, simply by allowing the index range on $\mu^{i}$ to be $0 \leq i \leq n$.

It should be emphasised that in writing the inverse metric as in (6.8), we are using an "overcomplete" basis of vector fields, since on $S^{n}$ we have $\frac{1}{2} n(n-1)$ Killing vectors $K^{i j}$ on a space that is only $n$-dimensional. The advantage of using this overcomplete basis, however, is that the expression (6.8) does not require the use of the $S^{n}$-dependent quantity $\widetilde{\Delta}=\widetilde{T}_{i j} \mu^{i} \mu^{j}$ that appears in the metric ansatz in (6.2).

The next step is to write the inverse metric (6.8) for the $S^{3}$ reduction in terms of self-dual and anti-self-dual combinations of the $S O(4)$ Killing vectors, since these are the combinations that generate the right and left actions of $S U(2)$. This will cast the $S^{3}$ inverse-metric ansatz into a form that admits a natural generalisation to the case of any group manifold $G$. The right and left combinations for $S^{3}$, denoted by $R^{a}$ and $L^{a}$, are given by

$$
R^{a}=\frac{1}{2} \eta_{i j}^{a} K^{i j}, \quad L^{a}=\frac{1}{2} \bar{\eta}_{i j}^{a} K^{i j}
$$

where $\eta_{i j}^{a}$ and $\bar{\eta}_{i j}^{a}$ are the self-dual and anti-self-dual 't Hooft tensors, whose components are defined by

$$
\eta_{0 b}^{a}=-\delta_{b}^{a}, \quad \eta_{b c}^{a}=-\epsilon_{a b c}, \quad \bar{\eta}_{0 b}^{a}=-\delta_{b}^{a}, \quad \eta_{b c}^{a}=\epsilon_{a b c} .
$$

Defining associated sets of right and left $S U(2)$ Yang-Mills fields by

$$
A_{\mu}^{a}=\frac{1}{4} \eta_{i j}^{a} A_{\mu}^{i j}, \quad B_{\mu}^{a}=\frac{1}{4} \bar{\eta}_{i j}^{a} A_{\mu}^{i j}
$$

we can rewrite $(6.8)$ as

$$
\begin{aligned}
\left(\frac{\partial}{\partial \hat{s}}\right)^{2}= & \frac{1}{2} g^{2} M_{a b} R^{a} \otimes R^{b}+\frac{1}{2} g^{2} N_{a b} L^{a} \otimes L^{b}+\frac{1}{2} g^{2} P_{a b}\left(R^{a} \otimes L^{b}+L^{b} \otimes R^{a}\right) \\
& +g^{\mu \nu}\left(\partial_{\mu}+g A_{\mu}^{a} R^{a}+g B_{\mu}^{a} L^{a}\right) \otimes\left(\partial_{\nu}+g A_{\nu}^{b} R^{b}+g B_{\nu}^{b} L^{b}\right)
\end{aligned}
$$

where $M_{a b}, N_{a b}$ and $P_{a b}$ are given in terms of $\widetilde{T}_{i j}$ by

$$
M_{a b}=\frac{1}{4} \widetilde{T}_{i l} \widetilde{T}_{j \ell} \eta_{i j}^{a} \eta_{k \ell}^{b}, \quad N_{a b}=\frac{1}{4} \widetilde{T}_{i l} \widetilde{T}_{j \ell} \bar{\eta}_{i j}^{a} \bar{\eta}_{k \ell}^{b}, \quad P_{a b}=\frac{1}{4} \widetilde{T}_{i l} \widetilde{T}_{j \ell} \eta_{i j}^{a} \bar{\eta}_{k \ell}^{b} .
$$

After some manipulations, we can show that the matrices $M$ and $N$ in (6.13) are related to the matrix $P$ by

$$
M=\left(1+P P^{t}\right)^{1 / 2}, \quad N=\left(1+P^{t} P\right)^{1 / 2},
$$


where $P^{t}$ denotes the transpose of $P$. Thus the scalar fields in the $S^{3}$ ansatz are parameterised purely by $P_{a b}$, which is in the representation $\left(\operatorname{Adjoint}\left(S U(2)_{L}\right)\right.$, $\left.\operatorname{Adjoint}\left(S U(2)_{R}\right)\right)$ of the $S U(2)_{L} \times S U(2)_{R}$ isometry group. This exact result is in accordance with the linearised scalar field analysis in [25].

We are now in a position to conjecture the form of the exact metric ansatz for the Pauli reduction of the bosonic string on any group manifold $G$. Namely, the proposal is that the inverse metric is given by the same expression (6.13), where now $R^{a}$ and $L^{a}$ are the Killing vectors associated with the right and left actions of $G$ on the group manifold. We again require also that (6.15) hold, so that the scalar fields will be parameterised just by $P_{a b}$, which is in the $\left(\operatorname{Adjoint}\left(G_{L}\right)\right.$, Adjoint $\left.\left(G_{R}\right)\right)$ representation of the group $G$. This is in accordance with the linearised results in [25], and indeed one can check that our conjectured exact ansatz (6.13) agrees after linearisation with the expression found in [25].

\section{Superpotentials and Solutions}

In this section, we return to the theme of DeWitt reductions of the bosonic string, which we explored in section 3 . We shall now look for some explicit domain-wall solutions of the flow equations in the DeWitt-reduced theories.

\subsection{Superpotentials for the truncated system}

We shall now examine the structure of the potential $V$ for the scalar fields in $n$ dimensions in more detail. To begin, it is convenient to set to zero all the scalars described by the unimodular matrix $h_{\alpha \beta}$ (meaning that we take $h_{\alpha \beta}=\delta_{\alpha \beta}$ ), and also to set to zero the

scalars $\widetilde{\chi}_{\alpha \beta}$ coming from $\hat{B}_{(2)}$. The truncation of these fields can easily be seen to be consistent, and the resulting potential $\widetilde{V}$ is given by

$$
\widetilde{V}=-\frac{1}{2} q e^{-\frac{1}{2} a \phi}\left(g^{2} e^{-\gamma \tilde{\varphi}}-\frac{1}{3} m^{2} e^{-3 \gamma \tilde{\varphi}}\right) .
$$

The system of gravity coupled to the two remaining scalars $\phi$ and $\tilde{\varphi}$ is itself a consistent truncation of (3.29), and is described by the $n$-dimensional Lagrangian

$$
\mathcal{L}=R * \mathbb{1}-\frac{1}{2} * d \phi \wedge d \phi-\frac{1}{2} * d \tilde{\varphi} \wedge d \tilde{\varphi}-\widetilde{V} * \mathbb{1}
$$

We can now seek a superpotential $W$, such that

$$
\widetilde{V}=\left(\frac{\partial W}{\partial \phi}\right)^{2}+\left(\frac{\partial W}{\partial \tilde{\varphi}}\right)^{2}-\frac{(n-1)}{2(n-2)} W^{2} .
$$


It should be noted that the coefficient multiplying $W^{2}$ here must necessarily have this specific value, if one is to be able to formulate first-order equations that solve the equations of motion following from (7.2). (See, e.g. [28, 29] and references therein.)

We can find three different choices for a superpotential $W$ such that (7.3) yields the truncated potential $\widetilde{V}$ given in (7.1) (modulo inessential sign choices). Each works only for a specific value of the dimension $q$ of the group manifold $G$ on which the bosonic string was reduced; these dimensions are $q=1,3$ and 25 , and so we shall denote the associated superpotentials by $W_{1}, W_{3}$ and $W_{25}$ respectively. They are given by

$$
\begin{aligned}
W_{1} & =\frac{q}{2 \sqrt{6}} e^{-\frac{1}{4} a \phi}\left(3 g^{2} m^{-1} e^{\frac{1}{2} \gamma \tilde{\varphi}}+m e^{-\frac{3}{2} \gamma \tilde{\varphi}}\right), \\
W_{3} & =\frac{q}{3 \sqrt{2}} e^{-\frac{1}{4} a \phi}\left(-3 g e^{-\frac{1}{2} \gamma \tilde{\varphi}}+m e^{-\frac{3}{2} \gamma \tilde{\varphi}}\right), \\
W_{25} & =\frac{q}{10 \sqrt{6}} e^{-\frac{1}{4} a \phi}\left(5 g e^{-\frac{1}{2} \gamma \tilde{\varphi}}-m^{2} g^{-1} e^{-\frac{5}{2} \gamma \tilde{\varphi}}\right) .
\end{aligned}
$$

The constant $\gamma$ is given by (3.30) with $q=1,3$ and 25 respectively. The case $q=1$ is vacuous, since there is no harmonic 3 -form in a 1-dimensional group manifold. We have no explanation, beyond the superficial calculational one, for why $q=3$ and $q=25$ should turn out to be only two cases where a superpotential leading to first-order equations exists.

\subsection{Brane solutions from superpotentials}

Using these superpotentials, we can obtain first-order equations for domain-wall configurations, and these equations can then be solved explicitly. To do this, we make the ansatz

$$
d s_{n}^{2}=e^{2 A} d x^{\mu} d x_{\mu}+d y^{2}
$$

where $A, \phi$ and $\tilde{\varphi}$ are assumed to depend only on the transverse coordinate $y$. From (7.2), the equations of motion are given by

$$
\begin{aligned}
\phi^{\prime \prime}+(n-1) A^{\prime} \phi^{\prime} & =\frac{\partial V}{\partial \phi}, \\
\tilde{\varphi}^{\prime \prime}+(n-1) A^{\prime} \tilde{\varphi}^{\prime} & =\frac{\partial V}{\partial \tilde{\varphi}}, \\
A^{\prime \prime}+(n-1) A^{\prime 2} & =-\frac{V}{n-2}, \\
\frac{1}{2} \phi^{\prime 2}+\frac{1}{2} \tilde{\varphi}^{2}-(n-1)(n-2) A^{\prime 2} & =V,
\end{aligned}
$$

where a prime denotes a derivative with respect to $y$. It is easy to see that these equations will be satisfied if the first-order equations

$$
\phi^{\prime}=\sqrt{2} \frac{\partial W}{\partial \phi}, \quad \tilde{\varphi}^{\prime}=\sqrt{2} \frac{\partial W}{\partial \tilde{\varphi}}, \quad A^{\prime}=-\frac{1}{\sqrt{2}(n-2)} W
$$


hold.

We find the following solutions, where, without loss of generality, we have set $g=m$ :

- $\underline{q=25:}$

Using $W=W_{25}$, we find that the first-order equations (7.7) admit the domain-wall solution

$$
\begin{aligned}
d s_{n}^{2} & =e^{2 A}\left(d x^{\mu} d x_{\mu}+H^{\frac{1}{2}} d r^{2}\right) \\
e^{\frac{1}{2} a \phi} & =e^{2 A}=\left(\frac{m^{2} r^{2}}{48}\right)^{25 /(n-2)} H^{5 /(2(n-2))}, \quad e^{\gamma \tilde{\varphi}}=\frac{1}{48} m^{2} r^{2} H^{\frac{1}{2}}
\end{aligned}
$$

where $\gamma=\sqrt{2} / 5$ and

$$
H=1+\frac{2304}{m^{4} r^{4}} .
$$

The solution (7.8) can be lifted back to $(n+q)$ dimensions using the DeWitt reduction ansätze of section 3, yielding

$$
\begin{aligned}
d \hat{s}^{2} & =H^{-10 /(n+23)} d x^{\mu} d x_{\mu}+H^{(n+3) /(2(n+23))}\left(d r^{2}+r^{2} d \Omega_{25}^{2}\right), \\
e^{\frac{1}{2} \hat{a} \hat{\phi}} & =H^{-10 /(n+23)}, \quad \hat{F}_{(3)}=\frac{1}{6} m f_{\alpha \beta \gamma} \sigma^{\alpha} \wedge \sigma^{\beta} \wedge \sigma^{\gamma} .
\end{aligned}
$$

where $d \Omega_{25}^{2}$ is the metric on the "unit" bi-invariant 25-dimensional group manifold $G$, scaled so that $R_{i j}=24 \delta_{i j}$. In terms of the left-invariant 1 -forms $\sigma^{\alpha}$, it is given by $d \Omega_{25}^{2}=$ $\frac{1}{48} g_{\alpha \beta} \sigma^{\alpha} \sigma^{\beta}$.

The lifted solution (7.10) has a singularity at $r=0$, which coincides with the horizon. Note that the 26-dimensional space transverse to the $(n-2)$-brane is nothing but the Ricci-flat cone over the bi-invariant group manifold $G$. Of course this transverse space also contributes its own power-law curvature singularity at $r=0$, at the apex of the cone. It is interesting to note, however, that in the string-frame metric, related to the Einstein-frame metric by

$$
d \hat{s}_{\text {string }}^{2}=e^{-\frac{1}{2} \hat{a} \hat{\phi}} d \hat{s}_{\text {Einstein }}^{2}
$$

the solution takes the form

$$
d \hat{s}_{\text {string }}^{2}=d x^{\mu} d x_{\mu}+H^{\frac{1}{2}}\left(d r^{2}+r^{2} d \Omega_{25}^{2}\right) .
$$

This metric is completely non-singular, since $H \sim 1 / r^{4}$ at small $r$. Thus the solution interpolates between $M_{n} \times G$ at small $r$, and $M_{n-1}$ times the Ricci-flat cone over $G$ at large $r$, where $M_{m}$ denotes $m$-dimensional Minkowski spacetime.

- $\underline{q=3:}$ 
The superpotential $W_{3}$ in (7.4) allows us to construct a domain wall in $n$ dimensions, given by

$$
\begin{aligned}
d s^{2} & =e^{2 A}\left(d x^{\mu} d x_{\mu}+H d r^{2}\right), \\
e^{\frac{1}{2} a \phi} & =e^{2 A}=\left(\frac{m^{2} r^{2}}{4}\right)^{3 /(n-2)} H^{1 /(n-2)}, \quad e^{\gamma \tilde{\varphi}}=\frac{1}{4} m^{2} r^{2} H,
\end{aligned}
$$

where $H=1+4 /\left(m^{2} r^{2}\right)$. Lifting back to $D=n+3$, and expressing the solution in the string frame, we recover the standard $(D-5)$-brane, given by

$$
\begin{aligned}
d \hat{s}^{2} & =d x^{\mu} d x_{\mu}+H\left(d r^{2}+d \Omega_{3}^{2}\right) \\
e^{\frac{1}{2} \hat{a} \hat{\phi}} & =H^{-2 /(n+1)}, \quad F_{(3)}=m \Omega_{(3)}
\end{aligned}
$$

where $d \Omega_{3}^{2}=\frac{1}{4} \sigma_{\alpha}^{2}$ is the bi-invariant metric on the unit 3 -sphere.

\subsection{Branes without superpotentials}

We showed in section 7.1 that in exceptional cases, namely when the group manifold has dimension $q=3$ or $q=25$, the scalar potential in the truncated $n$-dimensional theory (7.2) can be derived from a superpotential. In section 7.2 we made use of these superpotentials to construct explicit domain-wall solutions, which we then lifted back to the original $(n+q)$ dimensional bosonic string theory. In this section, we consider the generic situation when $q$ is equal to neither 3 nor 25 , in which case we have no option but to solve the secondorder equations (7.6) coming from (7.2). Since these equations are quite involved, we shall resort to approximate methods and numerical integration in order to study the form of the solutions. Before doing this, however, we shall consider a simplified exact solution in which the scalar field $\tilde{\varphi}$ is (consistently) set to zero.

Setting $g=m$, we can set $\tilde{\varphi}=0$ in (7.6), and then we can easily see that a solution is given by

$$
\begin{aligned}
d s_{n}^{2} & =e^{2 A} d x^{\mu} d x_{\mu}+d y^{2} \\
e^{\frac{1}{2} a \phi} & =e^{2 A}=\frac{q m^{2} y^{2}}{3(n-2)^{2}}
\end{aligned}
$$

Lifted to $(n+q)$ dimensions, this becomes

$$
\begin{aligned}
d \hat{s}^{2} & =\left(\frac{q m^{2} y^{2}}{3(n-2)^{2}}\right)^{(n-2) /(n+q-2)}\left[d x^{\mu} d x_{\mu}+m^{-2} g_{\alpha \beta} \sigma^{\alpha} \sigma^{\beta}+\frac{3(n-2)^{2}}{q m^{2} y^{2}} d y^{2}\right] \\
e^{\frac{1}{2} \hat{a} \hat{\phi}} & =\left(\frac{q m^{2} y^{2}}{3(n-2)^{2}}\right)^{(n-2) /(n+q-2)} \\
\hat{G}_{(3)} & =\frac{1}{6} m f_{\alpha \beta \gamma} \sigma^{\alpha} \wedge \sigma^{\beta} \wedge \sigma^{\gamma} .
\end{aligned}
$$


In the string-frame metric, defined in (7.11), the metric becomes simply

$$
d \hat{s}^{2}=d x^{\mu} d x_{\mu}+m^{-2} g_{\alpha \beta} \sigma^{\alpha} \sigma^{\beta}+\frac{3(n-2)^{2}}{q m^{2} y^{2}} d y^{2} .
$$

Note that $g_{\alpha \beta} \sigma^{\alpha} \sigma^{\beta}$ is just the bi-invariant metric on the group manifold $G$, with Ricci tensor given, in orthonormal components, by $R_{i j}=\frac{1}{2} \delta_{i j}$. In the string frame, the metric is totally regular; it is the direct sum of the metric on $n$-dimensional Minkowski spacetime and the bi-invariant metric on the group manifold $G$.

Another special case corresponds to taking $m=0$. The $n$-dimensional solution is then given by

$$
\begin{aligned}
d s_{n}^{2} & =e^{2 A}\left(d x^{\mu} d x_{\mu}+d r^{2}\right), \\
e^{\frac{1}{2} a \phi} & =e^{2 A}=\left(\frac{g^{2} r^{2}}{2(q-1)}\right)^{q /(n-2)}, \quad e^{\gamma \tilde{\varphi}}=\frac{g^{2} r^{2}}{2(q-1)},
\end{aligned}
$$

where $\gamma=\sqrt{2 / q}$. Lifted back to $(n+q)$ dimensions, it becomes

$$
\begin{aligned}
d \hat{s}^{2} & =d x^{\mu} d x_{\mu}+d r^{2}+r^{2} d \Omega_{q}^{2}, \\
\hat{\phi} & =0, \quad \hat{G}_{(3)}=0,
\end{aligned}
$$

where

$$
d \Omega_{q}^{2} \equiv \frac{1}{2(q-1)} g_{\alpha \beta} \sigma^{\alpha} \sigma^{\beta}
$$

is the "unit" bi-invariant metric on the group manifold $G$, whose Ricci tensor satisfies $R_{i j}=(q-1) \delta_{i j}$. Thus the $(n+q)$-dimensional solution in this special case is just the direct sum of the $(n-1)$-dimensional Minkowski metric and the Ricci-flat metric on the cone over $G$.

We shall now demonstrate that there exists a more general brane solution that interpolates between the solution (7.15) at short distance, and the solution (7.18) at large distance. This more general solution is the analogue of the explicit solutions that we found in section 7.2 for the special cases $q=3$ and $q=25$. Since the second-order equations are quite difficult to solve explicitly, we shall begin here by considering a Taylor expansion valid at short distances. We find that the metric takes the form

$$
d s_{n}^{2}=e^{2 A} d x^{\mu} d x_{\mu}+d y^{2}
$$

with

$$
\begin{aligned}
e^{\frac{1}{2} a \phi} & =e^{2 A}=\frac{q m^{2} y^{2}}{3(n-2)^{2}}\left(1+b_{2} z^{2}+\cdots\right), \\
e^{-\gamma \varphi} & =1+c z+c_{2} z^{2}+\cdots
\end{aligned}
$$


where

$$
\begin{aligned}
z & =y \frac{(n-2)[-q+\sqrt{q(q+24)}]}{2 q}, \\
b_{2} & =-\frac{3 q c^{2}}{2[q+24 n-48+\sqrt{q(q+24)}]}, \quad c_{2}=\frac{[-q-42+\sqrt{q(q+24)}] c^{2}}{2[-18-q+\sqrt{q(q+24)}]}
\end{aligned}
$$

and $c$ is an integration constant. We then apply numerical methods using the above smalldistance expansion to set initial data. Lifting back to $D=n+q$ dimensions, we find that for the solution to be free from singularities in the string frame, the constant $c$ has to be chosen so that $c \leq 0$. In the string frame, the metric interpolates between $M_{n} \times G$ at small distance, and $M_{n-1}$ times the Ricci-flat cone over $G$ at large distance.

\section{Discussion}

A traditional approach to dimensional reduction, which became popular in the 1980's, consisted of identifying a "ground state" of the higher-dimensional theory that is (locally) a product of a lower-dimensional spacetime $M$ and an internal (usually compact) space $K$. In other words, above each point $x$ in $M$ there was a copy of $K$, called the fibre, whose metric varied as one moved about $M$. One then considered small fluctuations around this background. If $K$ admitted isometries, then the fluctuations fell into representations of the isometry group. Usually, one focused attention on the "massless sector," which would comprise a finite subset of the infinite towers of "Kaluza-Klein modes." If the internal space $K$ admitted isometries, then the massless sector would be expected to include the Yang-Mills gauge bosons associated with the isometry group of $K$.

At the linear level, one can of course always truncate out the massive fields, since by definition there is no coupling between the various fields. At the full non-linear level, however, the question of whether such a truncation may be consistently performed can be a subtle one, since it might be that massless fields act as sources for the massive fields that were originally set to zero. There is no such problem in the case of a circle or torus reduction, or for a DeWitt group manifold reduction, since the "massless sector" is characterised by its invariance under the simply-transitive action of the isometry group of the general fully nonlinear ansatz. Note that in the DeWitt case the original ground state, with its bi-invariant metric on the group manifold $G$, has a larger isometry than that of the general ansatz, since it admits the Killing vectors $R_{a}$ of $G_{R}$ as well as the Killing vectors $L_{a}$ of $G_{L} \cdot{ }^{10}$

\footnotetext{
${ }^{10}$ Recall that left-invariant vector fields $L_{a}$ generate right translations, while right-invariant vector fields $R_{a}$ generate left translations.
} 
For the case of a Pauli reduction, where $K$ is the coset space $G / H$, there is no universal prescription for writing down a reduction ansatz that would guarantee the full complement of gauge bosons in a consistent reduction. Indeed, for a Pauli reduction of a generic theory it is guaranteed that no such ansatz exists; we have discussed in previous sections some of the exceptional cases where a consistent Pauli reduction is possible.

More generally, one can consider a dimensional reduction in which one makes no a priori assumptions about the nature of the internal space $K$; it may not necessarily be related to a group manifold or a coset space. The induced metric $g_{m n}(x, y)$ on $K$ will vary from point to point on the base manifold, but only within a finite-dimensional family, or modulus space $\mathcal{M}$, whose coordinates $\phi$ define scalar fields $\phi(x)$ on spacetime. The modulus space may contain privileged "ground-states" which are typically more symmetric than the general metric in $\mathcal{M}$. For example, in the case of a DeWitt group-manifold reduction, the modulus space $\mathcal{M}$ is the space of left-invariant metrics on the group $G$, and the privileged metrics are the bi-invariant metrics, which are unique up to a scale; the associated scalar field $\phi(x)$ is called the "breathing mode." In the case of Pauli coset reductions, $\mathcal{M}$ contains $G$-invariant ground-state metrics on $K=G / H$, but it also includes metrics that are not invariant under $G$. For a more general reduction, such as a Calabi-Yau reduction, $\mathcal{M}$ will contain no metrics invariant under any continuous group, and there may not be any naturally-selected "ground-state." 11

An often-used starting point is a metric ansatz of the form

$$
\begin{aligned}
d \hat{s}^{2}= & g_{m n}(\phi(x), y)\left(d y^{m}-K_{a}^{m}(y) A_{\mu}^{a}(x) d x^{\mu}\right)\left(d y^{n}-K_{b}^{n}(y) A_{\nu}^{b}(x) d x^{\nu}\right) \\
& +W(\phi(x), y)^{2} g_{\mu \nu}(x) d x^{\mu} d x^{\nu}
\end{aligned}
$$

where the $K_{a}^{m}(y)$ are the Killing vector fields (if any) of the internal space $K$ in its ground state, $A_{\mu}^{a}(x)$ are the gauge bosons associated with the group generated by these Killing vectors, and $W(\phi(x), y)$ is a "warp factor." The second term in (8.1) is the metric orthogonal to the fibres, and it is a conformal to a fixed metric $g_{\mu \nu}$ on the base $M .{ }^{12}$ If one wants the lower-dimensional metric $g_{\mu \nu}(x)$ to describe gravity in the Einstein conformal frame (i.e.

\footnotetext{
${ }^{11}$ Note however that, as we shall discuss later, no Calabi-Yau reduction, of any theory, can be expected to satisfy the strict requirement of consistency that we are requiring in this paper.

${ }^{12}$ Mathematically this structure is an example of a "conformal (pseudo)-Riemannian submersion." A Riemannian submersion in general has a metric of the form$$
d \hat{s}^{2}=g_{m n}(\phi(x), y)\left(d y^{m}-A_{\mu}^{m}(x, y) d x^{\mu}\right)\left(d y^{n}-A_{\nu}^{n}(x, y) d x^{\nu}\right)+g_{\mu \nu}(x) d x^{\mu} d x^{\nu} .
$$

The ansatz (8.1) differs from this in that the cross terms take a particular factorised form and in general a $y$-dependent conformal factor is inserted in front of the second term.
} 
with a canonical $\sqrt{-g} R$ Einstein-Hilbert action), then the warp factor should be chosen to be

$$
W=\left(\frac{\sqrt{\operatorname{det}\left(g_{m n}(\phi(x), y)\right)}}{\sqrt{\operatorname{det}\left(g_{m n}(y)\right)}}\right)^{-\frac{1}{n-2}},
$$

where the lower-dimensional spacetime has dimension $n$, and $g_{m n}(y)$ is any $x$-independent metric on $K$. This "fiducial" metric on $K$ is needed in order to ensure that $W$ is a scalar with respect to coordinate transformations of the $y^{m}$. A convenient choice is to take the fiducial metric to be the ground-state metric.

It is interesting to compare the ansatz (8.1) with the exact answer in known cases. First, consider a DeWitt reduction, whose metric ansatz is

$$
d \hat{s}^{2}=g_{a b}(x)\left(\lambda^{a}-A^{a}\right)\left(\lambda^{b}-A^{b}\right)+\left(\operatorname{det} g_{a b}(x)\right)^{-\frac{1}{n-2}} g_{\mu \nu}(x) d x^{\mu} d x^{\nu},
$$

where the conformal factor in the second term has been chosen so that the lower-dimensional metric is in the Einstein conformal frame. Since the left-invariant 1-forms can be written as $\lambda^{a}=\lambda_{m}^{a}(y) d y^{m}$, we can rewrite (8.4) in the form of (8.1), with

$$
g_{m n}(x, y)=g_{a b}(x) \lambda_{m}^{a}(y) \lambda_{n}^{b}(y), \quad K_{a}^{m}(y)=L_{a}^{m}, \quad W^{2}=\left(\operatorname{det} g_{a b}(x)\right)^{-\frac{1}{n-2}},
$$

where $L_{a}^{m}$ are the vector fields dual to $\lambda^{a}$; i.e. $L_{a}^{m} \lambda_{m}^{b}=\delta_{a}^{b}$. In other words $L_{a}^{m}$ are the Killing vectors of right-translations of the bi-invariant metric on $G$. If we compare with the ansatz (8.1), we see that the Killing vectors $K_{a}^{m}$ in this case coincide with half of the total isometries of the background, i.e. of the bi-invariant metric on $G$, and they are not Killing vectors of the generic deformation described by the ansatz (8.4). In fact in this case, one might have hoped to obtain an ansatz that included the gauge bosons associated both with $G_{R}$ and $G_{L}$, but the DeWitt ansatz includes only those associated with $G_{R}$. As we discussed in section 6.2, while a consistent reduction including the gauge bosons of $G_{L} \times G_{R}$ definitely cannot be consistent if one begins with a generic higher-dimensional theory, it is believed that for the special case of the $D$-dimensional bosonic string such a consistent reduction is possible. Of course this is really a Pauli reduction on $G$ viewed as the coset $(G \times G) / G$, rather than a DeWitt reduction on the group manifold $G$. Note, incidentally, that the "warp factor" $W^{2}$ in a DeWitt reduction is independent of the coordinates $y^{m}$ of the internal space.

We can also make a comparison between (8.1) and the metric ansatz given in (5.14) for the Pauli reduction of Einstein-Maxwell-dilaton gravity on $S^{2}$. A convenient way to do this is by choosing $y_{1}=\mu_{1}$ and $y_{2}=\mu_{2}$ as independent coordinates on $S^{2}$, with $\mu_{3}=\sqrt{1-y_{1}^{2}-y_{2}^{2}}$. 
It is straightforward to see from the definition of $D \mu^{i}$ that we shall have

$$
D \mu^{m}=d y^{m}-K_{i}^{m} A^{i}
$$

where $K_{i}=-\epsilon_{i j k} \mu^{j}\left(\partial / \partial \mu^{k}\right)$ are the Killing vectors on the ground-state 2-sphere. In terms of the unconstrained coordinates $y^{m}(m=1,2)$ we have

$$
K_{1}=\mu_{3} \frac{\partial}{\partial y_{2}}, \quad K_{2}=-\mu_{3} \frac{\partial}{\partial y_{1}}, \quad K_{3}=y_{2} \frac{\partial}{\partial y_{1}}-y_{1} \frac{\partial}{\partial y_{2}}
$$

Thus we can express the Pauli metric reduction (5.14) as

$$
d s_{D}^{2}=g_{m n}\left(d y^{m}-K_{i}^{m} A^{i}\right)\left(d y^{n}-K_{j}^{n} A^{j}\right)+(Y \Delta)^{\frac{1}{D-2}} d s_{D-2}^{2},
$$

where

$$
g_{m n}=\left(T^{-1}\right)_{m n}-\mu_{3}^{-1}\left(T^{-1}\right)_{m 3} y^{n}-\mu_{3}^{-1}\left(T^{-1}\right)_{n 3} y^{m}+\mu_{3}^{-2}\left(T^{-1}\right)_{33} y^{m} y^{n} .
$$

A straightforward calculation shows that $\operatorname{det}\left(g_{m n}\right)=\Delta /\left(\mu_{3}^{2} \operatorname{det}\left(T_{i j}\right)\right)=\Delta /\left(\mu_{3}^{2} Y\right)$, where as usual $\Delta=T_{i j} \mu^{i} \mu^{j}$. Note that for the "fiducial metric" corresponding to $T_{i j}=\delta_{i j}$, we have $\operatorname{det}\left(\bar{g}_{m n}\right)=\mu_{3}^{-2}$. Substituting into (8.3), we find that the warp factor is given by

$$
W^{2}=(Y \Delta)^{\frac{1}{D-2}}
$$

which is indeed the factor appearing in the Pauli reduction ansatz (8.8). Thus we have confirmed that the $S^{2}$ Pauli metric reduction ansatz is indeed of the general form given in (8.1). One can straightforwardly show that all the other known Pauli reduction examples, such as the $S^{3}$ reduction of the bosonic string, also fit the general form of the reduction ansatz (8.1). Note that in the Pauli coset reduction, by contrast to the DeWitt groupmanifold reduction, the warp factor $W^{2}$ depends on the coordinates $y^{m}$ of the internal space as well as on the coordinates $x^{\mu}$ on the base. For the reader's convenience, the gauge group structures of Kaluza, DeWitt and Pauli reductions are summarised in Table 1 below.

\begin{tabular}{|c|c|c|c|}
\hline & Kaluza & DeWitt & Pauli \\
\hline \hline Internal Space, $K$ & $U(1)$ & $G$ & $G / H$ \\
\hline Gauge Bosons & $U(1)$ & $G_{R}$ & $G_{R}$ \\
\hline Generic Isometries & $U(1)$ & $G_{L}$ & None \\
\hline Ground-state Isometries & $U(1)$ & $G_{L} \times G_{R}$ & $G_{R}$ \\
\hline
\end{tabular}

Table 1: The gauge bosons, isometries for generic scalar configurations, and isometries for the most symmetric "ground-state" configuration for the various dimensional reduction schemes. 
While (8.1) is a general proposal for the metric reduction ansatz, which appears to work in all known cases where a consistent reduction is possible, it is very far from providing a complete ansatz because it says nothing about the reduction of any matter fields. As we have already remarked, consistent Pauli reductions can never work starting from pure higher-dimensional gravity, and it is only in very exceptional cases that they can work even when matter is included. To see this more clearly, it is instructive to see what happens when one substitutes the general metric reduction ansatz (8.1) into the higher-dimensional Einstein equations. In practice this is rather complicated if one retains the scalar fields, but in fact the relevant points can be made adequately if we set the scalars to zero; i.e. we take $g_{m n}(\phi(x), y)$ to be the ground-state metric $g_{m n}(y)$ on $K$, and we take $W(\phi(x), y)=1$. If one then substitutes the ansatz into the lower-dimensional components of the higherdimensional equation $\hat{R}_{A B}=0$, one finds (see, for example, [31])

$$
R_{\mu \nu}-\frac{1}{2} R g_{\mu \nu}=\frac{1}{2} Y_{a b}\left(F_{\mu \rho}^{a} F_{\nu}^{b \rho}-\frac{1}{4} F_{\rho \sigma}^{a} F^{b \rho \sigma} g_{\mu \nu}\right)+\frac{1}{2} R(K) g_{\mu \nu}
$$

where $R_{\mu \nu}$ and $R$ are the lower-dimensional ( $y$-independent) Ricci tensor and scalar, and $R(K)$ is the Ricci scalar of the ground-state metric on the internal space $K$. In this example, where we are reducing pure Einstein gravity, $Y_{a b}=g_{m n} K_{a}^{m} K_{b}^{n}$. The general problem of inconsistency of the reduction can be seen from the fact that the product of Killing vectors in $Y_{a b}$ is in general $y$-dependent, and so (8.11) is not a consistent lower-dimensional Einstein equation. There is no problem for Kaluza or DeWitt reductions, since the product of Killing vectors in $Y_{a b}$ is then $y$-independent. For Pauli reductions on cosets, $Y_{a b}$ depends on $y$, and consistency is only achievable, if at all, when additional matter is included in the higher-dimensional theory whose reduction ansatz contributes additional terms in (8.11) that "conspire" to produce a $y$-independent prefactor $Y_{a b}$ for the uncontracted "stress tensor" $\left(F_{\mu \rho}^{a} F_{\nu}^{b \rho}-\frac{1}{4} F_{\rho \sigma}^{a} F^{b \rho \sigma} g_{\mu \nu}\right)$ in the analogue of (8.11). For example, in the $S^{7}$ reduction of eleven-dimensional supergravity, one gets additional contributions from the reduction ansatz for the 4 -form, leading to

$$
Y_{a b}=g_{m n} K_{a}^{m} K_{b}^{n}+\frac{1}{2 m^{2}} g^{m n} g_{p q} \nabla_{m} K_{a}^{p} \nabla_{n} K_{b}^{q},
$$

where $R_{m n}=(q-1) m^{2} g_{m n}$, with $q=7$. The combination in (8.12) is, in fact, $y$-independent on any sphere $S^{q}$. In [30], a discussion of the $S^{7}$ reduction was given. A more general analysis of related consistency issues appears in [31], including a discussion of why the restoration of the scalars, which of course would be necessary for a full discussion of consistency, could not resolve the above inconsistency in this particular sector. Thus finding, by virtue of some 
"conspiracy" involving additional matter contributions, that the tensor $Y_{a b}$ in the lowerdimensional components of the higher-dimensional Einstein equation is $y$-independent is a necessary, but not sufficient, condition for having a consistent reduction ansatz. In the light of these discussions, it seems very unlikely that consistency could ever be achieved for ground state manifolds $K$ with non-transitively acting isometry groups, regardless of of the choice of additional matter.

It is worth remarking that in the known cases of consistent Pauli reductions the difficulty in finding the reduction ansatz for the form fields is enormously greater than that for the metric reduction ansatz. (A striking example is provided by the $S^{7}$ reduction of elevendimensional supergravity, for which a proof of consistency is presented in [17]. Although explicit formulae for the metric reduction are given, there is no complete explicit result for the reduction of the 4 -form field.)

In this paper, we have concentrated on the discussion of Kaluza and DeWitt groupmanifold reductions, where consistency is guaranteed, and Pauli coset reductions, where consistency is achieved only in certain exceptional cases. Reduction ansätze are sometimes considered in more general cases where the internal space $K$ is inhomogeneous, admitting either an intransitively-acting group of isometries, or no continuous isometries at all. In the rare examples of consistent Pauli reductions, it is only as a consequence of very remarkable "conspiracies" between the properties of the original higher-dimensional theory, and the specific reduction manifold, that the consistency is achievable at all. It is evident that for an inhomogeneous internal space $K$, the likelihood of any such analogous conspiracies arising is rather remote. Thus it would seem to be highly unlikely that any kind of nontrivial consistent reduction is possible for any inhomogeneous internal space. For example, there would appear to be no reason to expect that a consistent reduction on a CalabiYau manifold $K$ would ever be consistent, if one tried to retain the set of massless scalars corresponding to the moduli of $K$, while setting the massive modes to zero. This is because one can expect that there will be non-linear couplings in the full untruncated theory in which powers of the massless scalars act as sources for the massive scalars that one is trying to set to zero. Thus, while we are not in a position to offer a cast-iron proof of the inconsistency in such a case, we can assert that the "burden of proof" lies with those who would claim that such reductions are consistent. ${ }^{13}$

\footnotetext{
${ }^{13}$ This is quite a different matter from the question of whether one can, to a very good approximation, neglect the effect of ignoring the massive modes and their non-linear massless source-terms in a discussion of low-energy effective physics in four-dimensional string compactifications. Here, we are addressing a mathematical issue of exact embeddability, not a physical question of approximate decoupling of massive
} 


\section{Acknowledgments}

M.C. (on sabbatical leave from the University of Pennsylvania) is supported in part by DOE grant DE-FG02-95ER40893, NATO linkage grant No. 97061, National Science Foundation Grant No. INT02-03585 and the Fay. R. and Eugene L. Langberg Chair. M.C. would like to thank the Univ. of Texas A\&M for hospitality during the initial stages of this work and the Institute for Advanced Study, Princeton, for hospitality and support during the course of this work. H.L and C.N.P. are supported in part by DOE grant DE-FG03-95ER40917. G.W.G. would like to than the Director and staff of the George P. and Cynthia W. Mitchell Institute for Fundamental Physics for their hospitality during the course of this work. C.N.P. would like to thank the Cambridge Relativity and Gravitation Group for hospitality during the completion of this work.

\section{A $\quad$ DeWitt $=$ Pauli $\circ$ DeWitt: Reductive Coset Reductions}

In this appendix, we derive explicit expressions for the DeWitt reduction on the group manifold $G$ can be viewed as an initial DeWitt reduction on the subgroup $H$, followed by a Pauli reduction on the coset $G / H$, in cases where the coset is reductive.

Using the appropriate definitions given in sections 5.2 and 5.3, we have

$$
\begin{aligned}
\rho^{a} T_{a} & =d h h^{-1}+h d k k^{-1} h^{-1} \\
& =\left(R^{\alpha}+\omega^{\alpha}\right) T_{\alpha}+\rho^{i} T_{i},
\end{aligned}
$$

where the $R^{\alpha}$ are right-invariant 1-forms on $H$,

$$
R^{\alpha} T_{\alpha}=d h h^{-1}
$$

and $h d k k^{-1} h^{-1}=\omega^{\alpha} T_{\alpha}+\rho^{i} T_{i}$. We also have

$$
\begin{aligned}
\lambda^{a} T_{a} & =k^{-1} h^{-1} d h k+k^{-1} d k=k^{-1}\left(\Lambda^{\alpha} T_{\alpha}\right) k+k^{-1} d k \\
& =T_{a} U^{a}{ }_{\alpha}\left(R^{\alpha}+\omega^{\alpha}\right)+T_{a} U^{a}{ }_{i} \rho^{i} \\
& =T_{a} U^{a}{ }_{\alpha} W_{\beta}{ }^{\alpha}\left(\Lambda^{\beta}+W^{\beta}{ }_{\gamma} \omega^{\gamma}\right)+T_{a} U^{a}{ }_{i} \rho^{i},
\end{aligned}
$$

where $\Lambda^{\alpha} T_{\alpha}=h^{-1} d h$ defines the left-invariant 1-forms on $H$, and $W^{\alpha}{ }_{\beta}$ is defined for $H$, in a manner analogous to the definition of $U^{a}{ }_{b}$ for $G$, by

$$
T_{\alpha} W_{\beta}^{\alpha}=h^{-1} T_{\beta} h
$$

modes. 
The next task is to show that the various terms in the final line of (A.3) are all independent of the $H$ subgroup coordinates $\tau_{\alpha}$ (aside from their appearance in the left-invariant 1-forms $\Lambda^{\alpha}$ themselves).

First, we note that

$$
\begin{aligned}
T_{a} U^{a}{ }_{\alpha} W_{\beta}{ }^{\alpha} & =V^{-1} T_{\alpha} V W_{\beta}{ }^{\alpha}=k^{-1} h^{-1} T_{\alpha} h k W_{\beta}{ }^{\alpha}=k^{-1} T_{\gamma} k W^{\gamma}{ }_{\alpha} W_{\beta}{ }^{\alpha} \\
& =k^{-1} T_{\beta} k,
\end{aligned}
$$

thus showing that $U^{a}{ }_{\alpha} W_{\beta}{ }^{\alpha}$ is independent of $\tau_{\alpha}$.

Next, we note from (A.1) that

$$
\begin{aligned}
d k k^{-1} & =\omega^{\alpha} h^{-1} T_{\alpha} h+\rho^{i} h^{-1} T_{i} h \\
& =\omega^{\alpha} W^{\beta}{ }_{\alpha} T_{\beta}+\rho^{i} h^{-1} T_{i} h .
\end{aligned}
$$

If the coset is reductive, meaning in particular that $[H, K]=K$, we see that $h^{-1} T_{i} h=$ $B_{i}{ }^{j} T_{j}$ for some ( $\tau_{\alpha}$-dependent) matrix $B_{i}{ }^{j}$. Since the assumed reductivity means that there are no $T_{\alpha}$ terms coming from $h^{-1} T_{i} h$, it follows that the manifest $\tau_{\alpha}$-independence of $d k k^{-1}$ implies that we can independently deduce that $\omega^{\alpha} W^{\beta}{ }_{\alpha}$ and $\rho^{i} h^{-1} T_{i} h$ are independent of the coordinates $\tau_{\alpha}$. Finally, from

$$
\begin{aligned}
T_{a} U^{a}{ }_{i} \rho^{i} & =V^{-1} T_{i} V \rho^{i}=k^{-1} h^{-1} T_{i} h k \rho^{i} \\
& =k^{-1}\left(\rho^{i} h^{-1} T_{i} h\right) k
\end{aligned}
$$

the $\tau_{\alpha}$-independence of $\rho^{i} h^{-1} T_{i} h$ allows us to deduce that $U^{a}{ }_{i} \rho^{i}$ is independent of $\tau_{\alpha}$.

It is now apparent that with $\lambda^{a}$ given by the final line of (A.3), i.e.

$$
\lambda^{a}=U^{a}{ }_{\alpha} W_{\beta}{ }^{\alpha}\left(\Lambda^{\beta}+W^{\beta}{ }_{\gamma} \omega^{\gamma}\right)+U^{a}{ }_{i} \rho^{i},
$$

we can re-express the standard DeWitt metric reduction (5.26), after completing the square on the $\Lambda^{\alpha}$ terms, as

$$
\begin{aligned}
d \hat{s}^{2}= & e^{2 \alpha \varphi} d s^{2}+e^{2 \beta \varphi} \Delta_{\alpha \beta}\left(h^{\alpha}+\Delta^{\alpha \gamma} T_{a c} M^{a}{ }_{\gamma} h^{c}\right)\left(h^{\beta}+\Delta^{\beta \delta} T_{b d} M^{b} h^{d}\right) \\
& +e^{2 \beta \varphi}\left(T_{a b}-\Delta^{\alpha \beta} T_{a c} T^{b d} M^{c}{ }_{\alpha} M^{d}{ }_{\beta}\right) h^{a} h^{b},
\end{aligned}
$$

where we have defined

$$
\begin{aligned}
h^{a} & \equiv U^{a}{ }_{i}\left(\rho^{i}-U_{b}^{i} A^{b}\right), \quad h^{\alpha} \equiv \Lambda^{\alpha}+W_{\beta}^{\alpha}\left(\omega^{\beta}-U_{a}^{\gamma} A^{a}\right), \\
M^{a}{ }_{\beta} & \equiv U_{\gamma}^{a}{ }_{\gamma} W_{\beta}{ }^{\gamma}, \quad \Delta_{\alpha \beta} \equiv T_{a b} M_{\alpha}^{a} M_{\beta}^{b},
\end{aligned}
$$


and $\Delta^{\alpha \beta}$ is the inverse of $\Delta_{\alpha \beta}$; i.e. $\Delta^{\alpha \beta} \Delta_{\beta \gamma}=\delta_{\gamma}^{\alpha}$. Since all the quantities in (A.9) are independent of $\tau_{\alpha}$, except for the left-invariant 1-forms $\Lambda^{\alpha}$ themselves, the expression (A.9) can now be viewed as a metric ansatz for a standard DeWitt reduction on the group manifold $H$ with left-invariant 1 -forms $\Lambda^{\alpha}$.

After performing this DeWitt reduction on $H$, we obtain a theory which can then undergo a consistent Pauli reduction on the coset space $G / H$.

\section{B Further Examples of Coset Reductions}

\section{B.1 The case of $(G \times G) / G$}

Any group may be considered as a coset of the product of the group with itself with respect to the diagonal subgroup. This fact plays an important role in string theory and so we will describe here some of the relevant geometry. For clarity, and consistency with our previous notation we take $G \times G$ as pairs $g=\left(g_{1}, g_{2}\right)$, with $g_{1}, g_{2} \in G$, with the usual multiplication rule, and the diagonal subgroup $H=G_{\text {diag }}$, as pairs $h=(u, u)$. We can, at least in the neighbourhood of the identity, write uniquely $G \times G=H K$,

$$
\left(g_{1}, g_{2}\right)=(u, u)\left(v, v^{-1}\right)
$$

with $h=(u, u)$ and $k=\left(v, v^{-1}\right)$. It follows that $g_{1}=u v, g_{2}=u v^{-1}$, and thus

$$
w=v^{2}=g_{2}^{-1} g_{1} \quad u=g_{1} v^{-1}
$$

Thus as long as the square root $\left(g_{2}^{-1} g_{1}\right)^{\frac{1}{2}}$ exists and is unique, we have a unique decomposition. This will be true as long as the exponential map is onto.

Defining $D g \equiv d g-g A$, where the gauge potentials $A$ of $G \times G$ are expressed as $A=\left(A_{1}, A_{2}\right)$, one has

$$
\begin{aligned}
g^{-1} D g & =k^{-1}\left(h^{-1} d h+d k k^{-1}-k A k^{-1}\right) k \\
& =\left(v, v^{-1}\right)^{-1}\left(\left(u^{-1} d u, u^{-1} d u\right)+\left(d v v^{-1},-v^{-1} d v\right)+\left(v A_{1} v^{-1}, v^{-1} A_{2} v\right)\right)\left(v, v^{-1}\right)
\end{aligned}
$$

and thus

$$
h^{-1} d h=\left(u^{-1} d u, u^{-1} d u\right)
$$

is along the fibre. We now need to project the remaining terms in (B.3) parallel and perpendicular to the orbits of the diagonal subgroup $H$, as discussed in section 5.3. To do this we need a metric on the group $G \times G$, and we take the Killing metric which of course 
coincides with the product of the Killing metrics on the two factors. Thus elements of the Lie algebra in $(\mathfrak{h}, \mathfrak{h})$ are parallel, whilst elements in $(\mathfrak{h},-\mathfrak{h})$ are perpendicular. We therefore have

$$
\begin{aligned}
& \left(d k k^{-1}-k A k^{-1}\right)_{\|} \\
& =\frac{1}{2}\left(d v v^{-1}-v^{-1} d v+v A_{1} v^{-1}+v^{-1} A_{2} v, d v v^{-1}-v^{-1} d v+v A_{1} v^{-1}+v^{-1} A_{2} v\right),
\end{aligned}
$$

and

$\left(d k k^{-1}\right)_{\perp}=\frac{1}{2}\left(d v v^{-1}+v^{-1} d v+v A_{1} v^{-1}-v^{-1} A_{2} v,-d v v^{-1}-v^{-1} d v-v A_{1} v^{-1}+v^{-1} A_{2} v\right)$.

The DeWitt metric reduction ansatz for this case can then be written in the standard way. If, for simplicity, we omit the scalars (which would not, in general, be a consistent thing to do), the metric is then given by

$d s^{2}=\operatorname{Tr}\left(u^{-1} d u+\omega+\frac{1}{2} v A_{1} v^{-1}+\frac{1}{2} v^{-1} A_{2} v\right)^{2}+\frac{1}{4} \operatorname{Tr}\left(d v v^{-1}+v^{-1} d v+v A_{1} v^{-1}-v^{-1} A_{2} v\right)^{2}$,

with

$$
\omega \equiv \frac{1}{2}\left(d v v^{-1}-v^{-1} d v\right)
$$

The round metric induced on the coset $(G \times G) / G$ itself is thus

$$
d s_{\text {Base }}^{2}=\frac{1}{2} \operatorname{Tr}\left(d v v^{-1}+v^{-1} d v\right)^{2} .
$$

Remarkably, this may be expressed entirely in terms of $w=v^{2}$, as

$$
d s_{\text {Base }}^{2}=\frac{1}{4} \operatorname{Tr}\left(w^{-1} d w w^{-1} d w\right) .
$$

We have arrived at the bi-invariant metric on $H$, as expected, even though $d v v^{-1}+v^{-1} d v$ is the sum of left-invariant and right-invariant one forms, and thus has a well defined transformation only under the adjoint action of $H$ on $H$, i.e. $v \longrightarrow U^{-1} v U$.

It is instructive to consider the special case of $H=S U(2)$. We set

$$
v=\left(\begin{array}{cc}
t+i z & x+i y \\
-x+i y & t-i z
\end{array}\right),
$$

and

$$
w=\left(\begin{array}{cc}
\mu_{0}+i \mu_{3} & \mu_{1}+i \mu_{2} \\
-\mu_{1}+i \mu_{2} & \mu_{0}-i \mu_{3}
\end{array}\right)
$$

One has

$$
\mu_{0}=t^{2}-x^{2}-y^{2}-z^{2}, \quad \mu_{i}=2 t x_{i},
$$


with $i=1,2,3$. The inverses are

$$
t= \pm \frac{1}{\sqrt{2}} \sqrt{1+\mu_{0}}, \quad x_{i}=\frac{\mu_{i}}{\sqrt{2\left(1+\mu_{0}\right)}} .
$$

\section{B.2 The case of $S^{n}=S O(n+1) / S O(n)$}

As a further example, we shall work through in detail the case of $S^{n}=S O(n+1) / S O(n)$. Since the inclusion of the gauge fields is straightforward, but adds little to the geometrical discussion that we wish to give here, we shall omit them in what follows.

We express an arbitrary element $g$ of $S O(n+1)$ as $g=h g$, with $h \in S O(n)$. It is convenient to write the generators of $S O(n+1)$ as $\Sigma_{A B}=-\Sigma_{B A}$, and to decompose the fundamental index as $A=(0, i)$, so that the generators of the $S O(n)$ subgroup are $\Sigma_{i j}$. In a matrix representation

$$
h=\exp \left(\begin{array}{cc}
\Lambda_{i j} & 0 \\
0 & 0
\end{array}\right)
$$

and

$$
k=\exp \left(\begin{array}{cc}
1 & \mathbf{b} \\
-\mathbf{b}^{t} & 0
\end{array}\right)=\left(\begin{array}{cc}
1-\frac{\mathbf{x x}^{t}}{1+x_{0}} & \mathbf{x} \\
-\mathbf{x}^{t} & x_{0}
\end{array}\right),
$$

where $\mathbf{x}=\mathbf{b} \frac{\sin b}{b}$ with $b=|\mathbf{b}|, x_{0}=\sqrt{1-\mathbf{x}^{2}}$ and $x^{2}=\mathbf{x}^{t} \mathbf{x}$. Note that if we take $x_{0}>0$, we cover one half of the sphere, whilst if we take $x_{0}<0$ we cover the other half. The left-invariant forms on the coset are

$$
k^{-1} d k=\left(\begin{array}{cc}
\frac{\mathbf{x} d \mathbf{x}^{t}-d \mathbf{x} \mathbf{x}^{t}}{1+x_{0}} & d \mathbf{x}-\frac{\mathbf{x} d x_{0}}{1+x_{0}} \\
-d \mathbf{x}^{t}+\frac{\mathbf{x}^{t} d x_{0}}{1+x_{0}} & 0
\end{array}\right),
$$

while the right-invariant one forms are

$$
d k k^{-1}=\left(\begin{array}{cc}
\frac{d \mathbf{x} \mathbf{x}^{t}-\mathbf{x} d \mathbf{x}^{t}}{1+x_{0}} & d \mathbf{x}-\frac{\mathbf{x} d x_{0}}{1+x_{0}} \\
-d \mathbf{x}^{t}+\frac{\mathbf{x}^{t} d x_{0}}{1+x_{0}} & 0
\end{array}\right) .
$$

We denote the left-invariant 1-forms on $S O(n)$ by $\left(h^{-1} d h\right)_{i j}=\sigma_{i j}$. The projection of $d k k^{-1}$ that is parallel to the $S O(n)$ fibres is given by

$$
\left(d k k^{-1}\right)_{i j} \equiv \omega_{i j}=\frac{x_{i} d x_{j}-x_{j} d x_{i}}{1+x_{0}}=-\left(k^{-1} d k\right)_{i j} .
$$

The projection perpendicular to the fibres is given by

$$
K_{i} \equiv\left(d k k^{-1}\right)_{0 i}=d x_{i}-\frac{x_{i} d x_{o}}{1+x_{0}}=-\left(k^{-1} d k\right)_{0 i}
$$

The bi-invariant metric on $S O(n+1)$ is given by

$$
d s^{2}=-\frac{1}{2} \operatorname{Tr}\left(g^{-1} d g\right)^{2}=-\frac{1}{2} \operatorname{Tr}\left(h^{-1} d h+d k k^{-1}\right)^{2} .
$$


Using the formulae above one gets

$$
d s^{2}=\frac{1}{2}\left(\sigma_{i j}+\omega_{i j}\right)^{2}+K_{i} K_{i},
$$

with

$$
K_{i} K_{i}=d x_{0}^{2}+d x_{i}^{2}
$$

which is the metric on the $S^{n}$ base of this principal $S O(n)$ bundle (i.e. the bundle of orthonormal frames over $S^{n}$ ). The 1-forms $\sigma_{i j}$ span the $S O(n)$ fibres and the 1 -forms $\omega_{i j}$ give the horizontal connection with respect to the Killing metric.

From the point of view of dimensional reduction, both the bundle (i.e. $S O(n+1)$ ) and the base (i.e. $S^{n}$ ) are Einstein spaces. Dimensional reduction of the Einstein action on $G=S O(n+1)$ gives $S O(n+1)$ Einstein-Yang-Mills theory coupled to scalars in the symmetric tensor representation of the adjoint. The "breathing mode" corresponds to the determinant of this symmetric tensor, which is an $S O(n+1)$ singlet. Because of the cosmological term in the bundle, this singlet scalar will have a potential. In an Einstein solution given by the Killing metric, one of the scalars are excited. Yang-Mills fields are present in this background, whose potentials are given precisely by the $\mathfrak{s o}(\mathfrak{n})$-valued oneforms $\omega_{i j}$.

\section{References}

[1] T. Kaluza, On the problem of unity in physics, Sitzunber. Preuss. Akad. Wiss. Berlin. Math. Phys. K1, 966 (1921).

[2] O. Klein, Quantum theory and 5-dimensional theory of relativity, Z. Phys. 37, 895 (1926).

[3] P. Jordan, Extension of projective relativity, Ann. der Phys., Lpz. 1, 219 (1947).

[4] Y. Thiry, On the regularity of gravitational and electromagnetic fields in unitary theories. I $\mathscr{G}$ II, C.R. Acad. Sci. Paris 226, 216 and 1881 (1948).

[5] W. Pauli, Wissenschaftlichter Briefwechsel, Vol. IV, Part II (1999), Springer-Verlag, edited by K.V. Meyenn.

[6] N. Straumann, On Pauli's invention of non-Abelian Kaluza-Klein theory in 1953, grqc/0012054. 
[7] L. O'Raifeartaigh and N. Straumann, Early history of gauge theories and Kaluza-Klein theories, with a glance at recent developments, hep-ph/9810524.

[8] O. Klein, in New theories in physics, International Institute of Intellectual Cooperation, Paris, 1939.

[9] D.J. Gross, Oscar Klein and gauge theory, hep-th/9411233.

[10] B.S. DeWitt, in Relativity, groups and topology, Les Houches 1963 (Gordon and Breach, 1964).

[11] M.J. Duff, B.E.W. Nilsson and C.N. Pope, Kaluza-Klein approach to the heterotic string, Phys. Lett. B163, 343 (1985).

[12] R. Kerner, Generalisation of the Kaluza-Klein theory for an arbitrary non-Abelian gauge group, Ann. Inst. H. Poincaré 9, 143 (1968).

[13] Y.M. Cho and P.G.O. Freund, Non-Abelian gauge fields as Nambu-Goldstone fields, Phys. Rev. D12, 1711 (1975).

[14] J. Scherk and J.H. Schwarz, How to get masses from extra dimensions, Nucl. Phys. B153, 61 (1979).

[15] S.W. Hawking, On the rotation of the universe, Mon. Not. Roy. Astr. Soc. 142, 129 (1969).

[16] G.E. Sneddon, Hamiltonian cosmology: a further investigation, J. Phys. Math. Gen. 9, 229 (1976).

[17] B. de Wit and H. Nicolai, The consistency of the $S^{7}$ truncation In $D=11$ Supergravity, Nucl. Phys. B281, 211 (1987).

[18] H. Nastase, D. Vaman and P. van Nieuwenhuizen, Consistency of the $A d S_{7} \times S^{4}$ reduction and the origin of self-duality in odd dimensions, Nucl. Phys. B581, 179 (2000), hep-th/9911238.

[19] M. Cvetič, H. Lü, C.N. Pope, A. Sadrzadeh and T.A. Tran, Consistent SO(6) reduction of type IIB supergravity on $S^{5}$, Nucl. Phys. B586, 275 (2000), hep-th/0003103.

[20] H. Lü and C.N. Pope, Exact embedding of $N=1, D=7$ gauged supergravity in $D=11$, Phys. Lett. B467, 67 (1999), hep-th/9906168. 
[21] M. Cvetič, H. Lü and C.N. Pope, Gauged six-dimensional supergravity from massive type IIA, Phys. Rev. Lett. 83, 5226 (1999), hep-th/9906221.

[22] H. Lü, C.N. Pope and T.A. Tran, Five-dimensional $N=4, S U(2) \times U(1)$ gauged supergravity from type IIB, Phys. Lett. B475, 261 (2000), hep-th/9909203.

[23] M. Cvetič, H. Lü and C.N. Pope, Four-dimensional $N=4$, SO(4) gauged supergravity from $D=11$, Nucl. Phys. B 574, 761 (2000), hep-th/9910252.

[24] M. Cvetič, H. Lü and C.N. Pope, Consistent Kaluza-Klein sphere reductions, Phys. Rev. D62, 064028 (2000), hep-th/0003286.

[25] M.J. Duff, B.E.W. Nilsson, C.N. Pope and N.P. Warner, Kaluza-Klein approach to the heterotic string. 2, Phys. Lett. B171 (1986) 170.

[26] C.G. Callan, E.J. Martinec, M.J. Perry and D. Friedan, Strings in background fields, Nucl. Phys. B262, 593 (1985).

[27] A. Salam and E. Sezgin, D=8 supergravity, Nucl. Phys. B258, 284 (1985).

[28] M. Cvetič, S. Griffies and S.J. Rey, Static domain walls in N=1 supergravity, Nucl. Phys. B381, 301 (1992), hep-th/9201007.

[29] M. Cvetič and H.H. Soleng, Supergravity domain walls, Phys. Rept. 282, 159 (1997), hep-th/9604090.

[30] M.J. Duff, B.E.W. Nilsson, C.N. Pope and N.P. Warner, On the consistency of the Kaluza-Klein ansatz, Phys. Lett. B149, 90 (1984).

[31] P. Hoxha, R.R. Martinez-Acosta and C.N. Pope, Kaluza-Klein consistency, Killing vectors, and Kähler spaces, Class. Quant. Grav. 17, 4207 (2000),hep-th/0005172. 